\title{
Lithogeochemistry of Intrusive Rocks in the Halo Porphyry Copper-Molybdenum Prospect, Northeast Cambodia
}

\author{
Seang Sirisokha ${ }^{1,2^{*}}$, Kotaro Yonezu${ }^{1}$, Thomas Tindell ${ }^{1}$, Tetsuya Nakanishi ${ }^{3}$, Koichiro Watanabe ${ }^{1}$, \\ Jocelyn Pelletier ${ }^{4}$ \\ ${ }^{1}$ Department of Earth Resources Engineering, Kyushu University, Fukuoka, Japan, \\ ${ }^{2}$ Department of Geo-Resources and Geotechnical Engineering, Institute of Technology of Cambodia, Phnom Penh, Cambodia \\ ${ }^{3}$ Kyushu University Museum, Kyushu University, Fukuoka, Japan \\ ${ }^{4}$ Geology Resources Department, Geoccop, Montreal, Canada \\ Email: *ssirisokha@mine.kyushu-u.ac.jp
}

How to cite this paper: Sirisokha, S., Yonezu, K., Tindell, T., Nakanishi, T., Watanabe, K. and Pelletier, J. (2019) Lithogeochemistry of Intrusive Rocks in the Halo Porphyry Copper-Molybdenum Prospect, Northeast Cambodia. Open Journal of Geology, 9, 342-363.

https://doi.org/10.4236/ojg.2019.97023

Received: June 4, 2019

Accepted: July 21, 2019

Published: July 24, 2019

Copyright $\odot 2019$ by author(s) and Scientific Research Publishing Inc. This work is licensed under the Creative Commons Attribution International License (CC BY 4.0).

http://creativecommons.org/licenses/by/4.0/ Open Access

\begin{abstract}
The Halo copper-molybdenum prospect is a porphyry system in Ratanakiri province, northeastern part of Cambodia. There is only one research was carried out on this prospect about geological mapping and short wave infrared (SWIR) spectroscopy on alteration mineral identification. The purpose of this research is to confirm the deposit type from previous Angkor Gold's report and find the centre of porphyry deposit based on characteristic of intrusive rocks at surface and subsurface, characteristics of the intrusive rocks and alteration lithogeochemistry of intrusive and volcanic rocks by using Pearce Element Ratio (PER) analysis. PER analysis was used to examine the nature and extend of the alteration halos in the porphyry Halo copper-molybdenum prospect. The intrusive rocks and volcanic rocks in Halo, range from diorite to granite (quartz feldspar porphyry) in composition as well as dacite to trachyandesite (andesite porphyry) in composition, respectively. They were formed in a subduction-related tectonic setting, likely a volcanic arc. Trace elements spider diagrams were normalized to primitive mantle display strong enrichment in large-ion lithophile elements such as $\mathrm{Rb}, \mathrm{Ba}$ and $\mathrm{K}$ and depletion in some high-field strength elements such as $\mathrm{Nb}$ and $\mathrm{Ti}$, suggesting magmas generated in a subduction-related tectonic setting. PER analysis indicates a moderate to high degree of sericite alteration of dacites, quartz feldspar porphyries, andesite porphyries and granodiorite porphyries. Moreover, three alterations in the Halo prospect such as potassic (secondary K-feldspar and biotite), phyllic (sericite) and propylitic (epidote) alteration were identified, which are consistent with petrography analysis. The potassic
\end{abstract}


alteration zone is vectoring center of the hydrothermal system which may represent the locus of mineralization. Therefore the geochemical signature of potassic alteration within the quartz feldspar porphyry, andesite porphyry and granodiorite porphyry with high grad of copper ranges up to $2670 \mathrm{ppm}$ and molybdenum ranges up to $5297 \mathrm{ppm}$ represents a character for further exploration in the Halo porphyry copper-molybdenum prospect.

\section{Keywords}

Cambodia, Hydrothermal Alteration, Halo, Porphyry Copper-Molybdenite Deposit, Pearce Element Ratio Analysis

\section{Introduction}

The Halo porphyry copper-molybdenum prospect is a porphyry system in Ratanakiri province, northeastern part of Cambodia. The province has a potential for porphyry-type deposits such as porphyry copper-molybdenum (Halo prospect, China Wall prospect) and porphyry copper-molybdenum-gold (Okalla prospect) (Figure 1(a); [1]). The Halo porphyry copper-molybdenum prospect lies $2 \mathrm{~km}$ southeast of a strike slip fault trending NE-SW, known as the PhumSyarung-Dok Yong Fault corridor. The Halo prospect is hosted by intermediate to felsic intrusive and volcanic rocks. However, detailed geochemical characteristics of rocks and alteration lithogeochemistry were not studied yet. This is the first study that focuses on the characteristics of the intrusive rocks (major elements, trace elements, and rare earth elements) to constrain petrogenesis and tectonic setting, and lithogeochemistry of intrusive and volcanic rocks to demonstrate elements transportation during hydrothermal alteration at the Halo prospect. The main purpose of this study is to confirm the deposit type based on the datafrom by present study and to find the centre of porphyry deposit in the Halo prospect.

\section{Regional Tectonic Setting and Ore Deposits}

Cambodia is located in the southern part of Indochina Terrane, mainland of Southeast Asia. The mainland SE Asia comprises several Gondwana-derived terranes including Indochina, South China, Sibumasu and West Myanmar Terranes, which assembled and amalgamated by subduction-collision processes during the Late Palaeozoic to Mesozoic (Figure 1(a); [2]). The Indochina Terrane is made up of several tectonic units, which host mineralization belts such as Truong Son Fold Belt (TSFB), Loei Fold Belt (LFB), Dalat-Kratie Belt (DKB) [3]. The Halo porphyry copper-molybdenum prospect in northeastern Cambodia lying on the DKB consists of Triassic to Cretaceous sedimentary rocks intruded by Cretaceous (125 - $75 \mathrm{Ma}$ ) volcano-plutonic rocks, which are overlain by Quaternary intraplate basalts. The DKB extends across the region from Cambodia to southern Vietnam and overlies the southern continuation of the TSFB and LFB 



Figure 1. (a) Map showing tectonic divisions of the Indochina Terrane including mineralization belt of Cambodia, Vietnam, Laos and Thailand modified after [7]; (b) Lithological map in the Halo prospect with samples location UTM 48N WGS84 [10].

(Figure 1(a); [4]). The Cretaceous belt is chronologically comparable to the plutono-volcanic rocks of the late stage of the Yanshanian Orogeny in SE China (140 - $65 \mathrm{Ma})$ [5]. Thus, this belt could have formed in a similar tectonic setting as a southern continuation of the Yanshanian belt, which was formed by the subduction of the Palaeo-Pacific Plate beneath the Eurasia Plate (including the Indochina Terrane) ([5] \& [6]). The ore deposits in the DKB are intrusion-related gold (Figure 1(a); e.g. Okvau, Snoul in Cambodia [7] and TienThuan in southern Vietnam [8]), porphyry copper-gold, skarn lead-zinc, sediment-hosted gold (e.g. North Kratie in Cambodia; [7] \& [9]), and porphyry copper-molybdenum, gold-silver epithermal veins over-printing porphyry copper-molybdenum-(gold) (Canada Wall and OkallaEast in Cambodia; [1]) systems.

\section{The Halo Porphyry Copper-Molybdenum Deposit}

The Halo prospect is located on the edge of a monzogranite pluton to the south which is covered with a prominent silica cap outcrops on hilltops in the area. The host rocks of the Halo prospect are composed of felsic and intermediate intrusive and volcanic rocks. The volcanic rocks were intruded by diorite, granodiorite, and a quartz feldspar porphyry stock, which brought copper and molybdenite mineralization in veins and veinlets in both the intrusive and volcanic units [1]. The quartz feldspar porphyry exposed on the surface is medium to coarse-grained with abundant disseminated sulfides and appears to be the intru- 
sion that brought in the molybdenum-copper mineralization. It is typically altered to silica, sericite, or sericite-chlorite [10]. Field observations of the core indicate that pyrite, molybdenite and chalcopyrite veinlets and veins exposed on surface exposures continue to the bottom of drill holes HD1 and HD2 (Figure 1(b)). Drill hole HD1 shows appreciable copper and silver mineralization in the top 99 metres, with anomalous levels of molybdenum corresponding to disseminated sulfides observed in the same interval. Included in the $99 \mathrm{~m}$ interval is an intercept of $2345 \mathrm{ppm} \mathrm{Cu}, 1.34 \mathrm{~g} / \mathrm{t} \mathrm{Ag}$, as well as $261.4 \mathrm{ppm}$ Mo over $88.9 \mathrm{~m}$ which includes $7.9 \mathrm{~m}$ of $8043 \mathrm{ppm} \mathrm{Cu}, 2.24 \mathrm{~g} / \mathrm{t} \mathrm{Ag}$, and $320.7 \mathrm{ppm}$ Mo from 10.1 to $18.0 \mathrm{~m}$ [11].

A major magnetic, concentrically ringed "doughnut" anomaly is present in the central part of the Halo porphyry copper-molybdenum prospect [10]. This magnetic anomaly is a typical expression of a porphyry system with a magnetic high in the center surrounded by a zone of lower magnetic response. The high magnetism typically represents an increase of the abundance of magnetite associated with the mineralization in the centre of porphyry type deposit, with surrounding low magnetism which reflects a depletion of the abundance of magnetite in the mineralized host rocks [12]. Termite mound geochemistry revealed anomalies of copper and molybdenum at the central part of Halo (Figure 1(b)) with the high up to $700 \mathrm{ppm} \mathrm{Cu}$ while the anomaly is generally $>250 \mathrm{ppm} \mathrm{Cu}$, covering $1.5 \mathrm{~km}$ extending WNW-ESE; $1 \mathrm{~km}$ extending NNE-SSW. High anomaly of molybdenum is $400 \mathrm{ppm}$ in $1.2 \mathrm{~km}$ by $75 \mathrm{~m}$, extending EW-NS from central part of Halo. Rock chip geochemistry ranges up to $7470 \mathrm{ppm} \mathrm{Cu}$ up to $1450 \mathrm{ppm}$ Mo at the central part of Halo (Figure 1(b); [1]). The main alteration zones developed in this prospect on the surface are inner phyllic (sericite), outer phyllic (sericite-chlorite), argillic (kaolinite-dickite-pyrophyllite-halloysite), propylitic (chlorite-epidote-quartz-calcite) and silicic alteration (quartz) according to field observation and short wave infrared spectroscopy on alteration mineral identification (Figure 2(a); [10]). Phyllic alteration is located the central part of the Halo prospect and was overprinted with silicic alteration (outcrop sample on the hilltops, up to $15 \mathrm{~m}$ ) [10].

\section{Analytical Methods}

Whole-rock major elements oxides, trace and rare earth elements concentrations of 42 samples including diorite, granodiorite, quartz feldspar porphyry, hornblende diorite collected from surface and four drill holes HD1, HD2, HD3 and HD4 (Figure 2(b)) were analyzed by X-ray fluorescence (XRF) spectroscopy using a RIGAKU RIX-3100 and by inductively coupled mass spectrometry (ICP-MS) Alilent Technologies 7500, respectively, at the Center of Advanced Instrumental Analysis, Kyushu University. Loss on ignition (LOI) was determined by heating the samples at $1000^{\circ} \mathrm{C}$ for 2 hours to determine relative weight loss. Thirty petrographic thin sections were prepared to identify textural characteristics and alteration minerals of rocks and observed using a Nikon Eclipse E600 


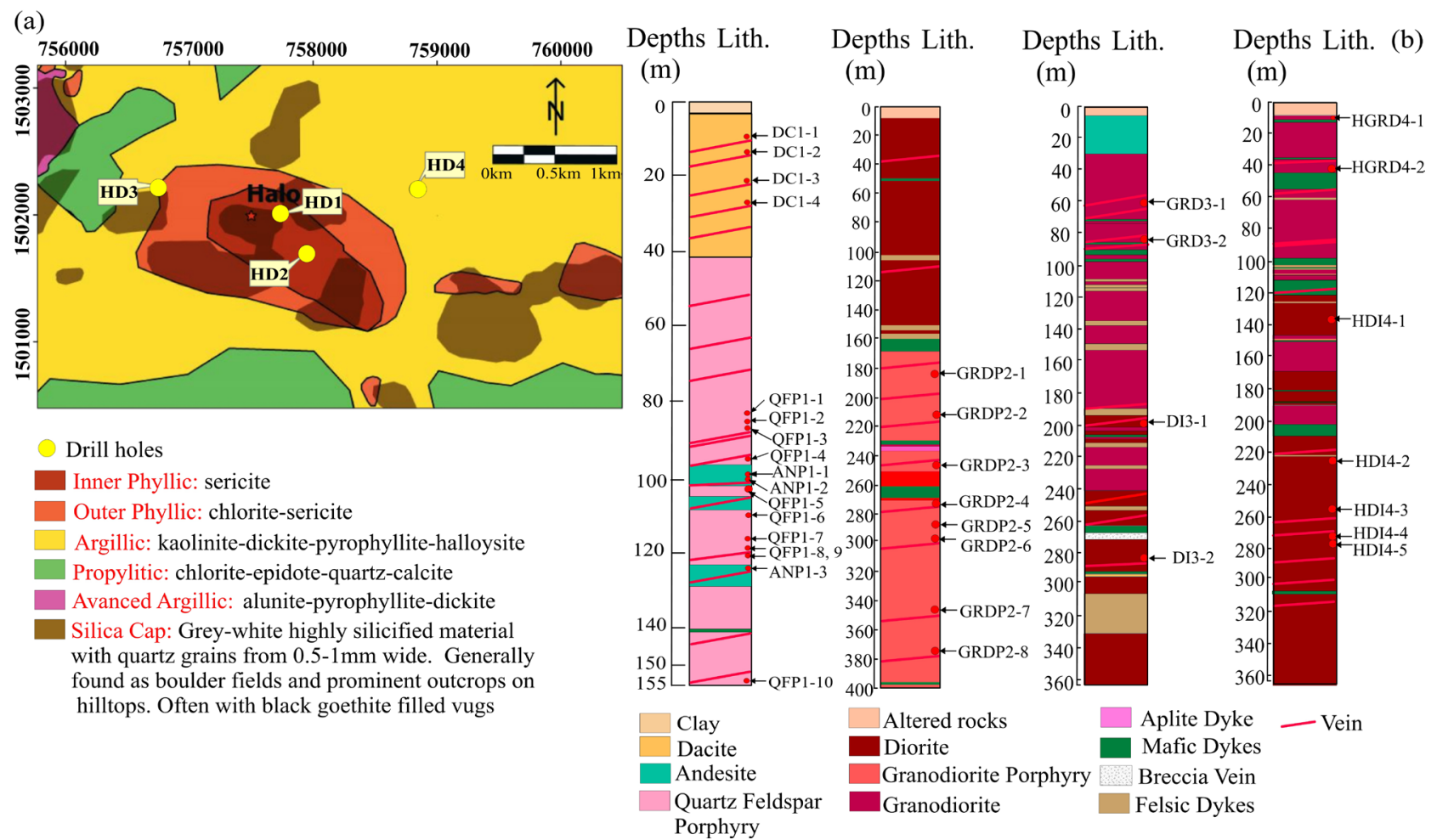

Figure 2. (a) Halo prospect alteration map from filed observation and SWIR interpretation [10]; (b) Core logs of four drill cores studies here (from left to right HD1, HD2, HD3 and HD4) showing the approximate position of samples.

POL microscope equipped with an AdvanCam-U3II camera. Both bulk and clay fraction X-ray diffraction analysis were conducted to identifyalteration mineral assemblage using a RigakuUltima IV X-ray diffractometer at Department of Earth Resources Engineering, Kyushu University.

\section{Lithology in the Halo Prospect}

In order to determine textural and mineral composition of each rock type, petrography was conducted on the relatively fresh samples, least altered samples and also altered samples including outcrop samples and drill core samples.

\subsection{Outcrop Samples}

In the central part of Halo, diorite, granodiorite, andesite and rhyolite were cut by faults trending NE-SW. In the southwestern part of Halo, andesite, rhyolitic tuff, granodiorite and syeno-granite were cut by strike slip fault trending NE-SW, known as PhumSyarung-Dok Yong Fault (Figure 1(b)). Diorite is dark-gray to greenish light gray in color and medium-grained, and consists mainly of plagioclase (up to $1.2 \mathrm{~mm}$ across), quartz (less than $1 \mathrm{~mm}$ across), $\mathrm{K}$-feldspar (up to $0.8 \mathrm{~mm}$ long) and biotite (less than $1 \mathrm{~mm}$ across). Granodiorite is dark-gray to greenish light gray in color and medium-grained, and consists mainly of anhedral to subhedral plagioclase (up to $1.4 \mathrm{~mm}$ across), quartz (less than $1.2 \mathrm{~mm}$ across), minor K-feldspar and biotite (Figures 3(A)-3(a)). 



Figure 3. Photographs (A)-(I) and photomicrographs (a)-(i) of selected igneous rocks, (A) and (a): granodiorite at surface; (B) and (b): dacite in HD1; (C) and (c): quartz feldspar porphyry in HD1; (D) and (d): andesite porphyry in HD1; (E), (e), (F) and (f): granodiorite porphyry in HD-2; (G) and (g): granodiorite in HD3; (H), (h), (I) and (i): hornblende diorite in HD4. Abbreviation: pl: plagioclase, Ksp: K-feldspar, hbl: hornblende, bt: biotite, qtz: quartz, ser: sericite, s.bt: secondary biotite, epi: epidote, py: pyrite and mag: magnetite, HD1-HD4: drill hole.

\subsection{Drill Core Samples}

Drill hole HD1 is composed mainly of dacite and andesite which were cut by numerous pink porphyry dykes (quartz feldspar porphyry), and fine-grained 
mafic dykes. The rocks in this drill hole are mostly affected by hydrothermal alteration. The majority of alteration in the top $85 \mathrm{~m}$ is phyllic alteration with patches of weak potassic alteration with magnetite. Alteration at the depth from 85 to $155 \mathrm{~m}$ is dominated by potassic alteration associated with stockworksveinlets, magnetite veinlet, quartz-magnetite-chalcopyrite veins, and quartz-pyritechalcopyrite-magnetite veins and dissemination of pyrite, chalcopyrite and magnetite. The dacite at the depth from 3 to $42 \mathrm{~m}$ is generally yellowish in color and associated with phyllic alteration that consists of sericite \pm kaolinite \pm chlorite based on petrography and XRD analysis (Figures 3(B)-3(b)). The quartz feldspar porphyry at the depth from 85 to $155 \mathrm{~m}$ is generally yellow to pink in color and medium to coarse-grained, and consists of euhedral to subhedralphenocrystic plagioclase (up to $8 \mathrm{~mm}$ across recognized in hand specimen), phenocrystic quartz (up to $2.8 \mathrm{~mm}$ ), groundmass mainly of granular quartz (less than $0.3 \mathrm{~mm}$ ), K-feldspar (less than $0.3 \mathrm{~mm}$ across), and biotite (up to $1.4 \mathrm{~mm}$ long), with accessory minerals that include zircon and $\mathrm{Fe}-\mathrm{Ti}$ oxides (mainly magnetite). The quartz feldspar porphyry is altered with feldspar replaced by sericite, biotite replaced by chlorite, while secondary K-feldspar occurs in groundmass (Figures 3(C)-3(c)). The andesite is pinkish dark green in color and associated with magnetite veinlets and $\mathrm{K}$-feldspar halo. Hydrothermal biotite occurs as aggregates of fine-grained flakes with quartz and magnetite (Figures 3(D)-3(d)).

Drill hole HD2 consists of diorite, granodiorite porphyry with patches of phyllic and weak potassic alteration. Mineralization in this drill hole occurs as quartz-molybdenite veins, quartz-pyrite-chalcopyrite veins, magnetite veinlets, quartz-chalcopyrite-pyrite-magnetite vein, pyrite veinlets and dissemination of pyrite and chalcopyrite. The granodiorite porphyry and the diorite were cut by fine mafic dykes, andesitic dykes, aplite dykes, and rhyolite dykes. The granodiorite is a dark-gray, pinkish dark-gray, pinkish light green in color, and medium to coarse-grained and consists of euhedral to subhedralphenocrystic plagioclase (up to $7 \mathrm{~mm}$ across), phenocrystic quartz (up to $1.6 \mathrm{~mm}$ ), K-feldspar (less than $0.3 \mathrm{~mm}$ ), biotite (less than $1.4 \mathrm{~mm}$ across), and hornblende (up to $1.2 \mathrm{~mm}$ long). Bitotite was partially altered to chlorite and secondary biotite (Figures 3(E)-3(f)).

Drill hole HD3 consists of sheared andesite at the top of the hole and transitioned to granodiorite cut by fine-grained mafic dykes. Alteration consists of phyllic and prophylitic alteration. Mineralization occurs as quartz-molybdenite veins, quartz-pyrite-chalcopyrite-molybdenite veins, quartz-magnetite-chalcopyrite-pyrite vein, quartz-pyrite-chalcopyrite-sphalerite-galena veins and disseminated pyrite and chalcopyrite. The granodiorite is light pinkish green in color, and medium-grained, and consists mainly of euhedral to subhedralphenocrystic plagioclase (up to $2.4 \mathrm{~mm}$ ), phenocrystic quartz (up to $1.4 \mathrm{~mm}$ ), and groundmass mainly of K-feldspar (less than $0.2 \mathrm{~mm}$ ) (Figures 3(G)-3(g)).

Drill hole HD4 is dominated by unaltered hornblende granodiorite and hornblende diorite, and transitioned into propylitic and phyllic altered diorite cut by massive polymetallic veins consisting dominantly of pyrite, quartz-cha- 
lcopyrite-sphalerite-galena veins and quartz-anhydrite-pyrite veins. The hornblende granodiorite and the hornblende diorite were cut by rhyolite dykes, and mafic dykes. The hornblende diorite is dark-gray in color, and medium-grained, and consists mainly of subhedral to euhedral plagioclase (up $2 \mathrm{~mm}$ across), euhedral to subhedral hornblende (up to $1.5 \mathrm{~mm}$ long), minor quartz (less than 1.2 $\mathrm{mm}$ across) that fill interstices between hornblende and plagioclase, and trace amount of biotite flakes (less than $0.8 \mathrm{~mm}$ across). The hornblende granodiorite is light pinkish gray in color, and medium-grained, and consists mainly of subhedral to euhedral plagioclase (up to $1 \mathrm{~mm}$ across), quartz (less than 1 $\mathrm{mm}$ across), K-feldspar (less than $0.6 \mathrm{~mm}$ long), euhedral to subhedral hornblende (up to $0.8 \mathrm{~mm}$ long). Some plagioclases were altered to epidote (Figures 3(H)-3(i)).

\section{Whole-Rock Major and Trace Elements Geochemistry}

Whole-rock compositions of 35 intrusive rocks and 7 volcanic rocks from the Halo prospect (Location of sample in Figure 1 and Figure 2) are determined in Table 1. The effects of hydrothermal alteration on whole-rock geochemical compositions have been assessed using a combination of petrography and bivariate plots (Figure 4(a)). Thus, samples from the Halo prospect were plotted on an alteration box plot diagram using the alteration index (AI) against the chlorite-carbonate-pyrite index (CCPI) proposed by [13]. The alteration index (AI) was calculated by 100 times of the ratio of the sum of $\mathrm{K}_{2} \mathrm{O}$ and $\mathrm{MgO}$ by the sum of $\mathrm{K}_{2} \mathrm{O}, \mathrm{MgO}, \mathrm{Na}_{2} \mathrm{O}$, and $\mathrm{CaO}$. The chlorite-carbonate-pyrite index (CCPI) were calculated by 100 times of the ratio of the sum of $\mathrm{MgO}$ and $\mathrm{FeO}$ by the sum of $\mathrm{MgO}, \mathrm{FeO}, \mathrm{Na}_{2} \mathrm{O}$, and $\mathrm{K}_{2} \mathrm{O}$. All major elements used to calculate $\mathrm{AI}$ and CCPI are expressed as wt.\%. The samples plotted on the alteration box plot diagram display 1) moderate K-feldspar-sericite, 2) sericite, 3) sericite-chlorite-pyrite, and 4) weak epidote-calcite \pm albite alteration trends. However, the majority of the samples do not significantly deviate from the least-altered box (Figure 4(a)). LOI (loss on ignition) values of least altered rocks range from $1.5 \%$ to $5.6 \%$, while LOI values of the altered rock range from $1.3 \%$ to $4.8 \%$. Thus the LOI values may misclassify between least altered and altered sample in this study. However, the result of least altered rocks classified by box plot [13] is consistent with the petrography. The $\mathrm{SiO}_{2}$ concentrations of intrusive rocks and volcanic rocks in the Halo prospect range from 57.7 to $75.6 \mathrm{wt} . \%$ and 61.7 to $68.2 \mathrm{wt} . \%$, respectively. The $\left(\mathrm{Na}_{2} \mathrm{O}+\mathrm{K}_{2} \mathrm{O}\right)$ versus $\mathrm{SiO}_{2}$ diagram [14] indicates that the intrusive rocks in the Halo are classified as diorites, granodiorites and granites (quartz feldspar porphyry) (Figure (4b)). The high field strength element (HFSE) bivariate plot of $\mathrm{Zr} / \mathrm{TiO} 2-\mathrm{Nb} / \mathrm{Y}$ [15] is used to distinguish geochemically the various volcanic rocks from the Halo prospect (Figure 5(a)). The volcanic rocks plot in rhyodacite/dacite to trachyandesite fields. The rocks classification are reliable for diorite and granodiorite as they are the least altered. The quartz feldspar porphyry spatially associated with mineralization, show strong alteration in box 
Table 1. Major (wt.\%), trace (ppm) and rare earth element (ppm) concentrations of intrusive and volcanic rocks.

(a)

\begin{tabular}{|c|c|c|c|c|c|c|c|c|c|c|}
\hline \multirow{2}{*}{$\begin{array}{c}\text { Depth } \\
\text { Samples ID }\end{array}$} & \multicolumn{6}{|c|}{ Outcrop samples } & \multirow{2}{*}{$\begin{array}{l}\text { D1-12 } \\
\text { DC1-1 }\end{array}$} & \multirow{2}{*}{$\begin{array}{l}\text { D1-17 } \\
\text { DC1-2 }\end{array}$} & \multirow{2}{*}{$\begin{array}{l}\text { D1-24 } \\
\text { DC1-3 }\end{array}$} & \multirow{2}{*}{$\begin{array}{l}\text { D1-28 } \\
\text { DC1-4 }\end{array}$} \\
\hline & DIO-1 & DIO-2 & DIO-3 & GRDO-1 & GRDO-2 & GRDO-3 & & & & \\
\hline Rock type & \multicolumn{3}{|c|}{ Diorite } & \multicolumn{3}{|c|}{ Granodiorite } & \multicolumn{4}{|c|}{ Dacite } \\
\hline Alteration & LA & LA & LA & LA & $\mathrm{A}$ & LA & A-PH & A-PH & A-PH & A-PH \\
\hline $\mathrm{SiO}_{2}$ (wt.\%) & 61.0 & 61.2 & 61.9 & 63.3 & 69.0 & 64.4 & 65.6 & 61.7 & 69.3 & 63.8 \\
\hline $\mathrm{TiO}_{2}$ & 0.8 & 0.9 & 0.8 & 0.7 & 0.6 & 0.6 & 0.5 & 0.5 & 0.5 & 0.5 \\
\hline $\mathrm{Al}_{2} \mathrm{O}_{3}$ & 15.7 & 16.3 & 15.1 & 15.6 & 14.3 & 15.7 & 18.0 & 18.0 & 15.1 & 15.7 \\
\hline $\mathrm{FeOT}$ & 6.8 & 6.3 & 6.0 & 5.7 & 3.0 & 5.7 & 2.0 & 4.3 & 2.4 & 4.5 \\
\hline $\mathrm{MnO}$ & 0.11 & 0.13 & 0.14 & 0.13 & 0.04 & 0.12 & 0.02 & 0.04 & 0.03 & 0.06 \\
\hline $\mathrm{MgO}$ & 4.3 & 4.4 & 3.8 & 3.2 & 2.2 & 3.2 & 1.3 & 2.5 & 1.6 & 2.8 \\
\hline $\mathrm{CaO}$ & 3.9 & 3.2 & 4.0 & 3.0 & 1.4 & 2.4 & 0.1 & 0.1 & 0.1 & 0.2 \\
\hline $\mathrm{Na}_{2} \mathrm{O}$ & 1.9 & 2.1 & 1.7 & 2.0 & 1.9 & 2.3 & 0.4 & 1.1 & 1.1 & 1.4 \\
\hline $\mathrm{K}_{2} \mathrm{O}$ & 2.1 & 2.2 & 2.8 & 2.4 & 4.2 & 2.0 & 7.2 & 5.0 & 6.3 & 4.9 \\
\hline $\mathrm{P}_{2} \mathrm{O}_{5}$ & 0.2 & 0.2 & 0.2 & 0.1 & 0.1 & 0.2 & 0.0 & 0.0 & 0.0 & 0.0 \\
\hline LOI & 2.6 & 2.7 & 2.4 & 2.8 & 2.7 & 3.2 & 4.1 & 4.9 & 2.3 & 4.0 \\
\hline Total & 99.5 & 99.6 & 99.0 & 99.0 & 99.3 & 99.8 & 99.2 & 98.2 & 98.9 & 97.8 \\
\hline $\mathrm{Cu}(\mathrm{ppm})$ & 80 & 60 & 250 & 80 & 413 & 61 & 3470 & 2333 & 4336 & 7048 \\
\hline Mo & n.d & n.d & n.d & n.d & 317 & n.d & 198 & n.d & 78 & 150 \\
\hline $\mathrm{Ti}$ & 4992 & 5134 & 4962 & 4118 & 3280 & 3702 & 3233 & 3078 & 2906 & 3031 \\
\hline $\mathrm{Ba}$ & 845 & 850 & 1038 & 926 & 910 & 432 & 1298 & 789 & 1133 & 774 \\
\hline $\mathrm{Sr}$ & 553 & 507 & 599 & 360 & 236 & 241 & 151 & 163 & 157 & 190 \\
\hline Y & 29 & 32 & 34 & 33 & 28 & 26 & 35 & 21 & 13 & 16 \\
\hline $\mathrm{Zr}$ & 225 & 231 & 252 & 157 & 164 & 155 & 216 & 202 & 223 & 146 \\
\hline $\mathrm{Rb}$ & 64 & 86 & 81 & 72 & 112 & 60 & 123 & 112 & 118 & 99 \\
\hline $\mathrm{Nb}$ & 7 & 7 & 8 & 5 & 5 & 5 & 9 & 8 & 8 & 6 \\
\hline $\mathrm{La}$ & 34.8 & 32.4 & 35.6 & 23.0 & 28.9 & 13.3 & 35.1 & 25.0 & 12.7 & 18.1 \\
\hline $\mathrm{Ce}$ & 81.1 & 75.2 & 81.9 & 52.6 & 64.7 & 27.3 & 76.9 & 47.2 & 25.8 & 35.4 \\
\hline $\operatorname{Pr}$ & 8.3 & 7.7 & 8.4 & 5.5 & 6.4 & 3.4 & 9.2 & 5.2 & 3.1 & 3.2 \\
\hline $\mathrm{Nd}$ & 36.2 & 33.7 & 36.3 & 24.7 & 27.7 & 12.8 & 32.6 & 17.2 & 10.3 & 14.6 \\
\hline $\mathrm{Sm}$ & 6.9 & 6.6 & 7.0 & 5.2 & 5.3 & 2.9 & 6.4 & 3.3 & 2.0 & 2.9 \\
\hline $\mathrm{Eu}$ & 1.81 & 1.81 & 1.83 & 1.36 & 1.24 & 0.74 & 1.70 & 0.87 & 0.60 & 0.65 \\
\hline Gd & 7.58 & 7.18 & 8.27 & 5.85 & 6.11 & 3.02 & 6.04 & 3.21 & 1.74 & 2.21 \\
\hline $\mathrm{Tb}$ & 0.73 & 0.66 & 0.77 & 0.59 & 0.62 & 0.53 & 0.88 & 0.48 & 0.27 & 0.36 \\
\hline Dy & 5.51 & 5.34 & 5.90 & 5.47 & 5.18 & 3.25 & 4.95 & 2.77 & 1.44 & 1.86 \\
\hline Ho & 1.04 & 0.99 & 1.10 & 1.03 & 0.99 & 0.68 & 0.96 & 0.51 & 0.30 & 0.35 \\
\hline Er & 3.31 & 3.03 & 3.34 & 3.26 & 3.20 & 2.16 & 2.64 & 1.49 & 0.89 & 1.04 \\
\hline $\mathrm{Tm}$ & 0.38 & 0.34 & 0.42 & 0.39 & 0.38 & 0.31 & 0.35 & 0.21 & 0.13 & 0.13 \\
\hline $\mathrm{Yb}$ & 3.26 & 2.96 & 3.78 & 3.13 & 3.37 & 2.10 & 2.35 & 1.43 & 0.96 & 0.83 \\
\hline $\mathrm{Lu}$ & 0.45 & 0.39 & 0.46 & 0.43 & 0.47 & 0.31 & 0.34 & 0.20 & 0.15 & 0.12 \\
\hline Total REE & 191.4 & 178.3 & 195.1 & 132.6 & 154.6 & 72.7 & 180.4 & 109.1 & 60.4 & 81.7 \\
\hline$(\mathrm{La} / \mathrm{Yb}) \mathrm{N}$ & 7.7 & 7.9 & 6.8 & 5.3 & 6.2 & 4.5 & 10.7 & 12.5 & 9.6 & 15.6 \\
\hline$(\mathrm{La} / \mathrm{Sm}) \mathrm{N}$ & 3.3 & 3.2 & 3.3 & 2.8 & 3.5 & 3.0 & 3.6 & 4.9 & 4.2 & 4.1 \\
\hline$(\mathrm{Gd} / \mathrm{Yb}) \mathrm{N}$ & 1.92 & 2.01 & 1.81 & 1.55 & 1.50 & 1.19 & 2.13 & 1.86 & 1.51 & 2.20 \\
\hline $\mathrm{Eu} / \mathrm{Eu}^{*}$ & 0.76 & 0.80 & 0.73 & 0.75 & 0.67 & 0.77 & 0.82 & 0.81 & 0.96 & 0.76 \\
\hline
\end{tabular}

Abbreviation: DIO: diorite on the surface, GRDO: granodorite on surface, DC1: dacite in drill hole HD1, LA: least altered, A-PH: phyllic alteration, n.d: not determined, number after D1 are presented the depth $(\mathrm{m})$. 
(b)

\begin{tabular}{|c|c|c|c|c|c|c|c|c|c|c|}
\hline Depth & D1-86 & D1-88 & D1-89 & D1-95 & D1-102 & D1-111 & D1-120 & D1-121 & $\mathrm{D}-123$ & D1-155 \\
\hline Samples ID & QFP1-1 & QFP1-2 & QFP1-3 & QFP1-4 & QFP1-5 & QFP1-6 & QFP1-7 & QFP1-8 & QFP1-9 & QFP1-10 \\
\hline Rock type & \multicolumn{10}{|c|}{ Quartz Feldspar Porphyry } \\
\hline Alteration & M-P & M-P & M-P & M-P & $\mathrm{M}-\mathrm{P}$ & M-P & $\mathrm{M}-\mathrm{P}$ & M-P & $\mathrm{M}-\mathrm{P}$ & $\mathrm{M}-\mathrm{P}$ \\
\hline $\mathrm{SiO}_{2}$ (wt.\%) & 72.4 & 72.9 & 74.5 & 71.4 & 74.3 & 75.6 & 69.7 & 73.1 & 69.2 & 73.4 \\
\hline $\mathrm{TiO}_{2}$ & 0.3 & 0.3 & 0.2 & 0.4 & 0.3 & 0.3 & 0.3 & 0.3 & 0.3 & 0.1 \\
\hline $\mathrm{Al}_{2} \mathrm{O}_{3}$ & 12.0 & 12.8 & 11.5 & 13.5 & 12.2 & 10.8 & 13.8 & 11.7 & 13.2 & 13.0 \\
\hline $\mathrm{FeOT}$ & 3.1 & 1.7 & 2.5 & 2.7 & 2.0 & 2.4 & 3.1 & 3.3 & 4.3 & 3.2 \\
\hline $\mathrm{MnO}$ & 0.02 & 0.01 & 0.02 & 0.04 & 0.03 & 0.01 & 0.10 & 0.03 & 0.03 & 0.03 \\
\hline $\mathrm{MgO}$ & 1.03 & 1.30 & 0.94 & 1.61 & 1.17 & 0.91 & 1.60 & 1.27 & 1.29 & 0.53 \\
\hline $\mathrm{CaO}$ & 0.09 & 0.12 & 0.10 & 0.21 & 0.14 & 0.11 & 0.13 & 0.12 & 0.11 & 0.07 \\
\hline $\mathrm{Na}_{2} \mathrm{O}$ & 0.95 & 1.15 & 1.08 & 1.66 & 1.46 & 1.11 & 1.48 & 1.22 & 1.40 & 0.81 \\
\hline $\mathrm{K}_{2} \mathrm{O}$ & 6.0 & 7.2 & 6.3 & 6.5 & 6.7 & 6.9 & 6.6 & 6.4 & 5.9 & 6.8 \\
\hline $\mathrm{P}_{2} \mathrm{O}_{5}$ & 0.02 & 0.03 & 0.02 & 0.04 & 0.02 & 0.01 & 0.02 & 0.02 & 0.02 & 0.00 \\
\hline LOI & 2.59 & 1.87 & 1.75 & 1.65 & 1.30 & 1.29 & 1.80 & 1.99 & 2.80 & 1.77 \\
\hline Total & 98.5 & 99.3 & 99.0 & 99.6 & 99.6 & 99.4 & 98.6 & 99.4 & 98.6 & 99.8 \\
\hline $\mathrm{Cu}(\mathrm{ppm})$ & 1536 & 2204 & 2432 & 650 & 769 & 1274 & 1669 & 514 & 682 & 152 \\
\hline Mo & 80 & 53 & 107 & 41 & n.d & n.d & 34 & n.d & n.d & n.d \\
\hline $\mathrm{Ti}$ & 1533 & 1777 & 1331 & 2306 & 1723 & 1575 & 2038 & 1919 & 1533 & 844 \\
\hline $\mathrm{Ba}$ & 797 & 929 & 916 & 1000 & 877 & 933 & 927 & 859 & 826 & 982 \\
\hline $\mathrm{Sr}$ & 90 & 174 & 92 & 222 & 177 & 102 & 141 & 126 & 118 & 62 \\
\hline $\mathrm{Y}$ & 10 & 6 & 9 & 13 & 6 & 6 & 7 & 4 & 5 & 4 \\
\hline $\mathrm{Zr}$ & 124 & 134 & 129 & 156 & 131 & 121 & 133 & 151 & 135 & 98 \\
\hline $\mathrm{Rb}$ & 117 & 123 & 112 & 111 & 109 & 111 & 129 & 118 & 122 & 128 \\
\hline $\mathrm{Nb}$ & 4 & 5 & 4.00 & 7.00 & 5 & 4 & 6 & 5 & 5 & 2 \\
\hline $\mathrm{La}$ & 11.1 & 9.0 & 10.2 & 11.8 & 8.6 & 4.3 & 8.9 & 5.4 & 6.6 & 4.6 \\
\hline $\mathrm{Ce}$ & 20.6 & 15.0 & 19.5 & 23.0 & 15.1 & 7.5 & 15.6 & 9.2 & 11.1 & 6.8 \\
\hline $\operatorname{Pr}$ & 2.3 & 1.7 & 2.3 & 2.8 & 1.7 & 0.8 & 1.7 & 0.9 & 1.3 & 0.8 \\
\hline $\mathrm{Nd}$ & 7.6 & 4.8 & 7.4 & 9.4 & 5.2 & 2.8 & 5.3 & 3.3 & 3.8 & 2.1 \\
\hline $\mathrm{Sm}$ & 1.48 & 0.93 & 1.36 & 1.85 & 1.01 & 0.49 & 0.99 & 0.55 & 0.77 & 0.42 \\
\hline $\mathrm{Eu}$ & 0.57 & 0.47 & 0.53 & 0.58 & 0.49 & 0.36 & 0.47 & 0.39 & 0.43 & 0.30 \\
\hline $\mathrm{Gd}$ & 1.50 & 0.76 & 1.22 & 1.80 & 0.93 & 0.51 & 0.81 & 0.60 & 0.68 & 0.33 \\
\hline $\mathrm{Tb}$ & 0.22 & 0.12 & 0.17 & 0.26 & 0.14 & 0.06 & 0.14 & 0.07 & 0.12 & 0.07 \\
\hline Dy & 1.15 & 0.55 & 0.90 & 1.42 & 0.75 & 0.36 & 0.78 & 0.35 & 0.61 & 0.37 \\
\hline Ho & 0.25 & 0.12 & 0.17 & 0.25 & 0.14 & 0.11 & 0.16 & 0.07 & 0.10 & 0.06 \\
\hline Er & 0.71 & 0.32 & 0.49 & 0.68 & 0.42 & 0.29 & 0.48 & 0.25 & 0.33 & 0.23 \\
\hline $\mathrm{Tm}$ & 0.08 & 0.03 & 0.06 & 0.09 & 0.05 & 0.04 & 0.07 & 0.04 & 0.04 & 0.04 \\
\hline $\mathrm{Yb}$ & 0.63 & 0.36 & 0.46 & 0.62 & 0.38 & 0.29 & 0.55 & 0.24 & 0.38 & 0.34 \\
\hline $\mathrm{Lu}$ & 0.09 & 0.05 & 0.07 & 0.09 & 0.06 & 0.05 & 0.09 & 0.05 & 0.06 & 0.06 \\
\hline Total REE & 48.3 & 34.1 & 44.8 & 54.7 & 35.0 & 17.9 & 36.0 & 21.3 & 26.4 & 16.5 \\
\hline$(\mathrm{La} / \mathrm{Yb}) \mathrm{N}$ & 12.60 & 17.80 & 15.90 & 13.70 & 16.00 & 10.80 & 11.50 & 16.20 & 12.30 & 9.60 \\
\hline$(\mathrm{La} / \mathrm{Sm}) \mathrm{N}$ & 4.80 & 6.30 & 4.80 & 4.10 & 5.50 & 5.70 & 5.80 & 6.30 & 5.50 & 7.10 \\
\hline$(\mathrm{Gd} / \mathrm{Yb}) \mathrm{N}$ & 1.96 & 1.75 & 2.21 & 2.40 & 1.99 & 1.48 & 1.21 & 2.10 & 1.46 & 0.79 \\
\hline $\mathrm{Eu} / \mathrm{Eu}^{*}$ & 1.15 & 1.67 & 1.23 & 0.96 & 1.51 & 2.19 & 1.55 & 2.07 & 1.77 & 2.41 \\
\hline
\end{tabular}

Abbreviation: QFP1: quartz feldspar porphyry in drill hole HD1, M-P: moderate potassic alteration, n.d: not determined, number after D1 are presented the depth $(\mathrm{m})$. 
(c)

\begin{tabular}{|c|c|c|c|c|c|c|c|c|c|c|c|}
\hline Depth & D1-99 & D1-104 & D1-126 & D2-187 & D2-218 & D2-248 & D2-273 & D2-294 & $\mathrm{D} 2-313$ & D2-356 & D2-280 \\
\hline Samples ID & ANP1-1 & ANP1-3 & ANP1-2 & GRDP2-1 & GRDP2-2 & GRDP2-3 & GRDP2-4 & GRDP2-5 & GRDP2-6 & GRDP2-7 & GRDP2-8 \\
\hline Rock type & \multicolumn{3}{|c|}{ Andesite Porphyry } & \multicolumn{8}{|c|}{ Granodiorite Porphyry } \\
\hline Alteration & $\mathrm{M}-\mathrm{P}$ & M-P & M-P & W-P & W-P & W-P & W-P & W-P & W-P & W-P & W-P \\
\hline $\mathrm{SiO}_{2}$ (wt.\%) & 65.0 & 68.2 & 62.4 & 66.9 & 71.5 & 65.5 & 66.3 & 65.4 & 67.5 & 65.8 & 66.3 \\
\hline $\mathrm{TiO}_{2}$ & 0.5 & 0.5 & 0.8 & 0.4 & 0.3 & 0.4 & 0.4 & 0.5 & 0.4 & 0.4 & 0.4 \\
\hline $\mathrm{Al}_{2} \mathrm{O}_{3}$ & 15.5 & 14.8 & 15.4 & 11.3 & 11.8 & 14.3 & 13.7 & 14.8 & 13.8 & 14.1 & 14.1 \\
\hline $\mathrm{FeOT}$ & 4.7 & 3.1 & 6.8 & 1.7 & 1.9 & 2.5 & 2.7 & 3.5 & 3.0 & 3.4 & 2.9 \\
\hline $\mathrm{MnO}$ & 0.04 & 0.06 & 0.07 & 0.02 & 0.06 & 0.03 & 0.10 & 0.05 & 0.04 & 0.06 & 0.05 \\
\hline $\mathrm{MgO}$ & 1.9 & 2.1 & 2.9 & 1.6 & 1.0 & 1.7 & 1.4 & 2.0 & 1.5 & 1.6 & 1.6 \\
\hline $\mathrm{CaO}$ & 0.3 & 0.1 & 0.7 & 3.6 & 4.1 & 2.9 & 3.4 & 3.0 & 3.2 & 3.0 & 3.2 \\
\hline $\mathrm{Na}_{2} \mathrm{O}$ & 2.1 & 1.7 & 2.1 & 1.5 & 1.8 & 2.1 & 1.9 & 2.4 & 2.1 & 2.0 & 2.1 \\
\hline $\mathrm{K}_{2} \mathrm{O}$ & 7.4 & 7.1 & 6.0 & 4.8 & 3.2 & 4.5 & 4.2 & 3.6 & 4.3 & 5.0 & 4.2 \\
\hline $\mathrm{P}_{2} \mathrm{O}_{5}$ & 0.1 & 0.0 & 0.2 & 0.1 & 0.1 & 0.1 & 0.1 & 0.1 & 0.1 & 0.1 & 0.1 \\
\hline LOI & 1.9 & 2.1 & 2.1 & 5.6 & 3.8 & 4.4 & 4.4 & 3.6 & 3.0 & 3.5 & 3.8 \\
\hline Total & 99.3 & 99.7 & 99.4 & 97.4 & 99.5 & 98.4 & 98.6 & 98.8 & 98.9 & 98.9 & 98.8 \\
\hline $\mathrm{Cu}(\mathrm{ppm})$ & 2003 & 399 & 371 & 239 & 383 & 558 & 332 & 578 & 481 & 251 & 767 \\
\hline Mo & n.d & n.d & n.d & n.d & n.d & n.d & 46 & n.d & n.d & n.d & n.d \\
\hline $\mathrm{Ti}$ & 3096 & 3084 & 4582 & 2127 & 1741 & 2228 & 2234 & 2662 & 2270 & 2413 & 2442 \\
\hline $\mathrm{Ba}$ & 1038 & 957 & 719 & 943 & 1007 & 931 & 953 & 882 & 958 & 1164 & 1018.0 \\
\hline $\mathrm{Sr}$ & 307 & 197 & 283 & 337 & 221 & 355 & 325 & 487 & 460 & 333.00 & 470.00 \\
\hline $\mathrm{Y}$ & 11 & 8 & 14 & 20 & 20 & 18 & 19 & 21 & 18 & 19 & 18.00 \\
\hline $\mathrm{Zr}$ & 134 & 152 & 198 & 133 & 137 & 158 & 170 & 181 & 169 & 150 & 176.00 \\
\hline $\mathrm{Rb}$ & 120 & 124 & 111 & 94 & 87 & 97 & 109 & 81 & 98 & 108 & 98.00 \\
\hline $\mathrm{Nb}$ & 7 & 8 & 11 & 9 & 6 & 6 & 6 & 7 & 6 & 6 & 6.00 \\
\hline $\mathrm{La}$ & 10.8 & 8.6 & 9.3 & 71.4 & 35.7 & 25.8 & 33.5 & 25.5 & 33.2 & 35.1 & 29.9 \\
\hline $\mathrm{Ce}$ & 20.6 & 15.7 & 19.3 & 116.3 & 58.1 & 48.0 & 59.9 & 47.9 & 61.7 & 54.1 & 54.3 \\
\hline $\operatorname{Pr}$ & 3.2 & 1.8 & 2.4 & 12.9 & 6.8 & 5.2 & 5.7 & 4.5 & 6.0 & 5.5 & 5.3 \\
\hline $\mathrm{Nd}$ & 9.1 & 5.8 & 8.4 & 36.0 & 23.1 & 15.9 & 20.3 & 17.5 & 21.4 & 18.6 & 19.4 \\
\hline $\mathrm{Sm}$ & 2.4 & 1.1 & 1.9 & 5.5 & 4.0 & 2.8 & 3.4 & 3.1 & 3.7 & 3.0 & 3.4 \\
\hline $\mathrm{Eu}$ & 1.41 & 0.46 & 0.59 & 1.24 & 0.82 & 0.82 & 0.85 & 0.71 & 0.92 & 0.75 & 1.02 \\
\hline $\mathrm{Gd}$ & 2.33 & 1.02 & 1.71 & 5.66 & 3.67 & 2.51 & 3.26 & 2.57 & 3.31 & 2.68 & 3.50 \\
\hline $\mathrm{Tb}$ & 1.03 & 0.17 & 0.26 & 0.62 & 0.47 & 0.34 & 0.42 & 0.38 & 0.46 & 0.34 & 0.48 \\
\hline Dy & 2.20 & 0.88 & 1.64 & 2.93 & 2.40 & 1.81 & 2.25 & 2.12 & 2.39 & 1.74 & 2.32 \\
\hline Ho & 1.15 & 0.18 & 0.32 & 0.55 & 0.46 & 0.38 & 0.43 & 0.42 & 0.47 & 0.34 & 0.49 \\
\hline Er & 1.96 & 0.50 & 0.92 & 1.77 & 1.48 & 1.10 & 1.41 & 1.31 & 1.49 & 1.12 & 1.51 \\
\hline $\mathrm{Tm}$ & 1.08 & 0.12 & 0.07 & 0.22 & 0.20 & 0.15 & 0.19 & 0.18 & 0.22 & 0.15 & 0.22 \\
\hline $\mathrm{Yb}$ & 1.76 & 0.52 & 0.84 & 1.30 & 1.33 & 1.09 & 1.30 & 1.24 & 1.38 & 0.98 & 1.37 \\
\hline $\mathrm{Lu}$ & 1.04 & 0.08 & 0.13 & 0.21 & 0.21 & 0.18 & 0.21 & 0.20 & 0.23 & 0.17 & 0.23 \\
\hline Total REE & 60.0 & 36.9 & 47.8 & 256.4 & 138.8 & 106.1 & 133.1 & 107.7 & 136.8 & 124.5 & 123.3 \\
\hline$(\mathrm{La} / \mathrm{Yb}) \mathrm{N}$ & 4.40 & 11.98 & 7.97 & 39.30 & 19.22 & 16.96 & 18.52 & 14.79 & 17.24 & 25.81 & 15.58 \\
\hline$(\mathrm{La} / \mathrm{Sm}) \mathrm{N}$ & 2.87 & 4.94 & 3.22 & 8.44 & 5.82 & 6.04 & 6.34 & 5.27 & 5.78 & 7.57 & 5.70 \\
\hline$(\mathrm{Gd} / \mathrm{Yb}) \mathrm{N}$ & 1.10 & 1.63 & 1.69 & 3.59 & 2.28 & 1.90 & 2.08 & 1.72 & 1.98 & 2.27 & 2.11 \\
\hline $\mathrm{Eu} / \mathrm{Eu}^{*}$ & 1.79 & 1.27 & 1.00 & 0.67 & 0.65 & 0.93 & 0.76 & 0.74 & 0.78 & 0.79 & 0.90 \\
\hline
\end{tabular}

Abbreviation: ANP1: andesite porphyries in drill hole HD1, GRDP2: granodiorite porphyry in HD2, W-P: weak potassic alteration, M-P: moderate potassic alteration, n.d: not determined, number after D1 and D2 are presented the depth (m). 
(d)

\begin{tabular}{|c|c|c|c|c|c|c|c|c|c|c|c|}
\hline Depth & D3-189 & D3-284 & D3-78 & D3-94 & $\mathrm{D} 4-39$ & D4-224 & D4-271 & D4-276 & D4-277 & D4-12 & D4-46 \\
\hline Samples ID & DI3-1 & DI3-2 & GRD3-1 & GRD3-2 & HDI4-1 & HDI4-2 & HDI4-3 & HDI4-4 & HDI4-5 & HGRD4-1 & HGRD4-2 \\
\hline Rock type & \multicolumn{2}{|c|}{ Diorite } & \multicolumn{2}{|c|}{ Granodiorite } & \multicolumn{5}{|c|}{ Hornblende diorite } & \multicolumn{2}{|c|}{$\begin{array}{l}\text { Hornblende } \\
\text { granodiorite }\end{array}$} \\
\hline Alteration & LA & LA & LA & $\mathrm{A}-\mathrm{PH}$ & LA & A-EP & LA & LA & LA & LA & LA \\
\hline $\mathrm{SiO}_{2}$ (wt.\%) & 57.8 & 62.4 & 69.9 & 66.0 & 62.2 & 62.2 & 59.5 & 58.2 & 59.4 & 63.0 & 68.4 \\
\hline $\mathrm{TiO}_{2}$ & 0.6 & 0.6 & 0.4 & 0.5 & 0.5 & 0.5 & 0.7 & 0.7 & 0.7 & 0.6 & 0.4 \\
\hline $\mathrm{Al}_{2} \mathrm{O}_{3}$ & 16.1 & 15.1 & 12.9 & 15.2 & 13.1 & 11.5 & 15.6 & 16.4 & 16.2 & 15.4 & 14.1 \\
\hline $\mathrm{FeOT}$ & 6.1 & 4.9 & 2.1 & 3.8 & 6.5 & 5.9 & 7.4 & 7.3 & 6.1 & 5.7 & 3.5 \\
\hline $\mathrm{MnO}$ & 0.49 & 0.14 & 0.44 & 0.45 & 0.18 & 0.15 & 0.17 & 0.18 & 1.13 & 0.13 & 0.13 \\
\hline $\mathrm{MgO}$ & 3.1 & 3.1 & 1.5 & 2.4 & 2.4 & 1.7 & 3.1 & 3.6 & 4.6 & 2.8 & 1.9 \\
\hline $\mathrm{CaO}$ & 4.5 & 3.4 & 2.7 & 2.2 & 9.2 & 11.9 & 7.6 & 7.3 & 4.7 & 5.1 & 3.3 \\
\hline $\mathrm{Na}_{2} \mathrm{O}$ & 3.0 & 2.6 & 1.4 & 1.2 & 1.5 & 1.3 & 1.9 & 2.1 & 2.6 & 2.4 & 2.9 \\
\hline $\mathrm{K}_{2} \mathrm{O}$ & 1.8 & 2.6 & 4.8 & 4.1 & 1.5 & 0.6 & 1.7 & 2.0 & 1.6 & 2.6 & 3.0 \\
\hline $\mathrm{P}_{2} \mathrm{O}_{5}$ & 0.15 & 0.14 & 0.07 & 0.07 & 0.15 & 0.15 & 0.18 & 0.18 & 0.20 & 0.15 & 0.14 \\
\hline LOI & 5.6 & 4.2 & 3.5 & 3.7 & 2.6 & 3.9 & 1.9 & 1.9 & 1.6 & 2.0 & 1.8 \\
\hline Total & 99.2 & 98.9 & 99.6 & 99.5 & 99.8 & 99.7 & 99.7 & 99.7 & 98.8 & 99.8 & 99.6 \\
\hline $\mathrm{Cu}(\mathrm{ppm})$ & 150 & 20 & 250 & 173 & 24 & 15 & 85 & 49 & 68 & 30 & 124 \\
\hline Mo & 48 & 12 & 214 & 72 & n.d & n.d & n.d & n.d & n.d & n.d & n.d \\
\hline $\mathrm{Ti}$ & 3364 & 3417 & 2104 & 2864 & 3209 & 3132 & 3952 & 3934 & 3976 & 3488 & 2335 \\
\hline $\mathrm{Ba}$ & 295 & 584 & 1037 & 790 & 272 & 165 & 619 & 723 & 411 & 716 & 968 \\
\hline $\mathrm{Sr}$ & 347 & 295 & 140 & 137 & 1079 & 1382 & 523 & 457 & 383 & 385 & 340 \\
\hline $\mathrm{Y}$ & 29 & 23 & 21 & 23 & 27 & 25 & 26 & 22 & 26 & 27 & 20 \\
\hline $\mathrm{Zr}$ & 83 & 131 & 136 & 155 & 128 & 143 & 117 & 126 & 93 & 128 & 135 \\
\hline $\mathrm{Rb}$ & 71 & 104 & 124 & 143 & 24 & 13 & 40 & 44 & 56 & 72 & 64 \\
\hline $\mathrm{Nb}$ & 5 & 5 & 5 & 6 & 5 & 5 & 4 & 4 & 4 & 6 & 6 \\
\hline $\mathrm{La}$ & 15.9 & 14.2 & 25.0 & 14.8 & 18.1 & 20.8 & 13.8 & 14.5 & 14.7 & 16.1 & 17.3 \\
\hline $\mathrm{Ce}$ & 31.7 & 27.6 & 45.4 & 28.6 & 37.1 & 41.0 & 28.6 & 29.3 & 31.3 & 32.8 & 34.0 \\
\hline $\operatorname{Pr}$ & 3.6 & 3.0 & 4.1 & 3.2 & 3.7 & 4.1 & 3.4 & 3.8 & 4.0 & 4.1 & 3.9 \\
\hline $\mathrm{Nd}$ & 14.2 & 11.8 & 16.1 & 12.0 & 16.8 & 17.5 & 13.9 & 13.0 & 14.7 & 14.6 & 12.2 \\
\hline $\mathrm{Sm}$ & 3.2 & 2.5 & 3.0 & 2.7 & 3.7 & 3.7 & 3.4 & 3.0 & 3.3 & 3.6 & 2.5 \\
\hline $\mathrm{Eu}$ & 0.71 & 0.65 & 0.84 & 0.72 & 0.87 & 0.81 & 1.15 & 1.12 & 0.74 & 0.97 & 0.78 \\
\hline $\mathrm{Gd}$ & 2.73 & 2.25 & 2.77 & 2.47 & 3.33 & 3.49 & 3.25 & 3.17 & 3.31 & 3.49 & 2.35 \\
\hline $\mathrm{Tb}$ & 0.53 & 0.42 & 0.44 & 0.50 & 0.62 & 0.63 & 0.71 & 0.71 & 0.52 & 0.61 & 0.37 \\
\hline Dy & 3.22 & 2.47 & 2.44 & 2.77 & 3.76 & 3.75 & 3.30 & 3.36 & 3.24 & 3.72 & 2.46 \\
\hline Ho & 0.68 & 0.52 & 0.51 & 0.62 & 0.81 & 0.76 & 0.82 & 0.90 & 0.68 & 0.83 & 0.48 \\
\hline $\mathrm{Er}$ & 2.12 & 1.60 & 1.63 & 1.84 & 2.51 & 2.39 & 2.25 & 2.35 & 2.09 & 2.36 & 1.43 \\
\hline $\mathrm{Tm}$ & 0.30 & 0.23 & 0.23 & 0.32 & 0.36 & 0.34 & 0.46 & 0.55 & 0.28 & 0.38 & 0.21 \\
\hline $\mathrm{Yb}$ & 2.06 & 1.54 & 1.58 & 1.77 & 2.40 & 2.34 & 2.14 & 2.22 & 2.08 & 2.21 & 1.55 \\
\hline $\mathrm{Lu}$ & 0.32 & 0.24 & 0.26 & 0.34 & 0.36 & 0.35 & 0.46 & 0.52 & 0.29 & 0.37 & 0.23 \\
\hline Total REE & 81.2 & 68.9 & 104.4 & 72.7 & 94.5 & 101.9 & 77.6 & 78.5 & 81.2 & 86.1 & 79.6 \\
\hline$(\mathrm{La} / \mathrm{Yb}) \mathrm{N}$ & 5.54 & 6.63 & 11.39 & 5.97 & 5.42 & 6.39 & 4.63 & 4.67 & 5.07 & 5.22 & 7.99 \\
\hline$(\mathrm{La} / \mathrm{Sm}) \mathrm{N}$ & 3.19 & 3.65 & 5.34 & 3.54 & 3.14 & 3.62 & 2.66 & 3.09 & 2.87 & 2.88 & 4.53 \\
\hline$(\mathrm{Gd} / \mathrm{Yb}) \mathrm{N}$ & 1.10 & 1.21 & 1.46 & 1.15 & 1.15 & 1.24 & 1.25 & 1.18 & 1.32 & 1.30 & 1.25 \\
\hline $\mathrm{Eu} / \mathrm{Eu}^{*}$ & 0.71 & 0.82 & 0.88 & 0.84 & 0.74 & 0.68 & 1.05 & 1.10 & 0.68 & 0.82 & 0.98 \\
\hline
\end{tabular}

Abbreviation: DI3 and GRD3: diorite and granodiorite in HD3, HDI4 and HGRD4: hornblende diorite and hornblende granodiorite in drill hole HD4, LA: Least altered, A-PH: phyllic alteration, A-EP: propylitic alteration, n.d: not determined, number after D3 and D4 are presented the depth (m). 


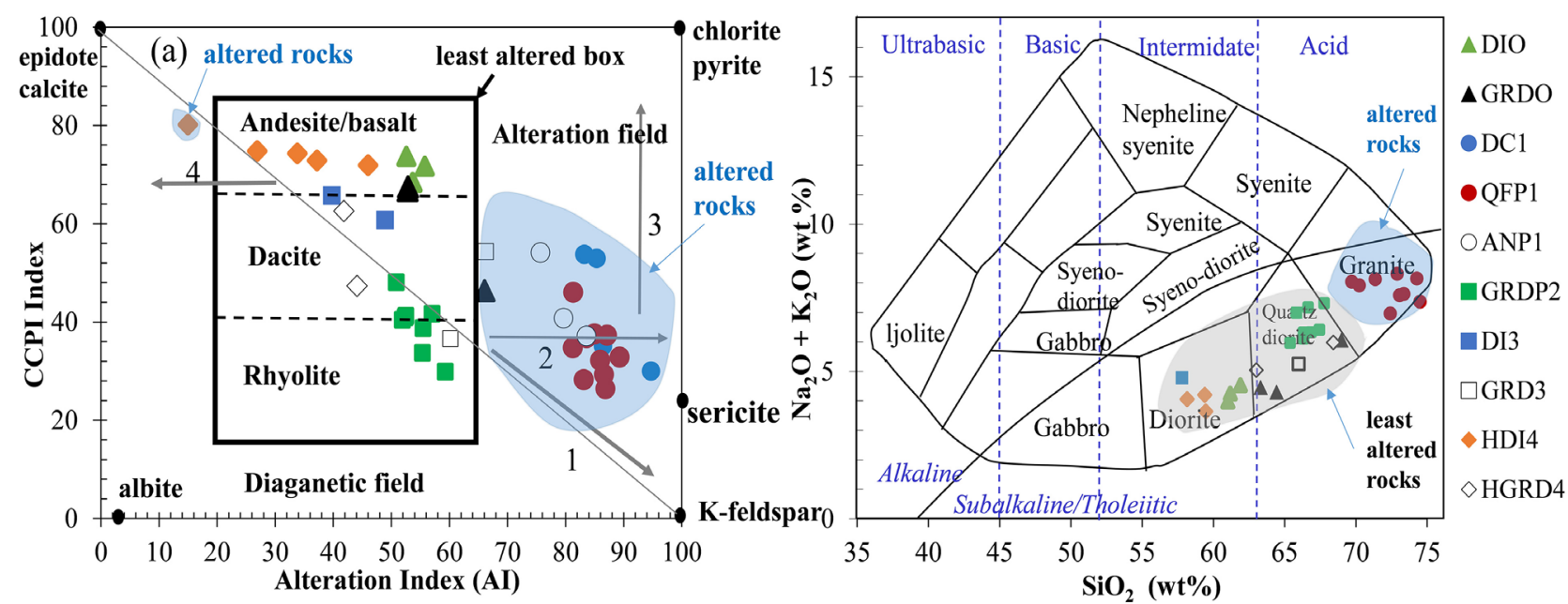

Figure 4. Alteration box plot with the alteration index (AI) versus the chlorite-carbonate-pyrite index (CCPI) [13]; (b) TAS diagram for plutonic rocks using total alkalis versus silica of [14]. Abbreviation: DIO: diorite on the surface; GRDO: granodorite on surface; QFP1: quartz feldspar porphyry in drill hole HD1, GRDP2: granodiorite porphyry in HD2; DI3 and GRD3: diorite and granodiorite, respectively, in HD3, HDI4 and HGRD4: hornblende diorite and hornblende granodiorite, respectively, in drill hole HD4.



(a)

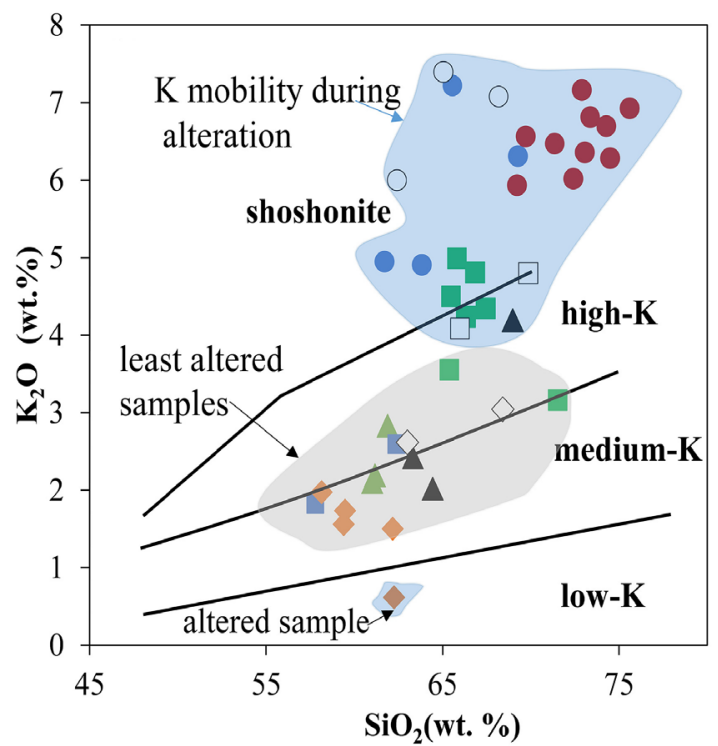

(b)

Figure 5. (a) $\mathrm{Zr} / \mathrm{TiO}_{2}$ versus $\mathrm{Nb} / \mathrm{Y}$ diagram derived for volcanic rocks of [15]; (b) $\mathrm{SiO}_{2}$ versus $\mathrm{K}_{2} \mathrm{O}$ (wt.\%) diagram of [16]. Abbreviation: DC1and ANP1: dacite and andesite porphyry, respectively, in drill hole HD1 and other symbols are the same as in Figure 4.

plots. Therefore, major elements are deemed unreliable to classify the rock types as strongly altered.

On the $\mathrm{SiO}_{2}$ versus $\mathrm{K}_{2} \mathrm{O}$ discrimination diagram for intrusive and volcanic rocks (Figure 5(b)), intrusive rocks are medium-K calc-alkaline, high-K calc-alkaline and shoshonitic fields [16]. The samples with high-K values contain hydrother- 
mal K-feldspar and biotite, implying that $\mathrm{K}$ was added to the rocks during hydrothermal alteration (potassic alteration). Thus, it is interpreted that the high- $\mathrm{K}$ trend is a result of potassic alteration and does not reflect primary igneous composition (e.g. shoshonite). The least altered granodiorite (GRDP2) plots in high-K calc-alkaline to shoshonite. Therefore, the primary igneous compositions of rock belong to the high-K calc-alkaline series. The least altered diorite and granodiorite exposed on surface plot in medium-K to high-K calc-alkaline field. Thus the primary composition of diorite and granodiorite belongs to medium- $\mathrm{K}$ calc-alkaline series. Diorite and granodiorite (DI3 and GRD3) plot on boundary between medium-K and high-K calc-alkaline field. Least altered hornblende diorite (HDI4) plots in medium-K calc-alkaline field except one sample with strong alteration plot in low-K calc-alkaline filed. The primary igneous compositions of the hornblende diorite belong to the high-K calc-alkaline series. In contrast, low-K composition is also affected by hydrothermal alteration. The granodiorite (HGRD4) plots on boundary between medium-K to high-K calc-alkaline, is close to primary igneous rock compositions. Based on the box plot and $\mathrm{SiO}_{2}$ versus $\mathrm{K}_{2} \mathrm{O}$ (wt.\%) diagram, some samples are strongly affected by $\mathrm{K}$ mobility. Therefore, we further use Pearce element ratio (PER) analysis to discuss in next section in order to elucidate about alteration lithogeochemistry [17].

Chondrite-normalized REE patterns [18] of the intrusive and volcanic rock from the Halo prospect (diorite, granodiorite, granodiorite porphyry, quartz feldspar porphyry, dacite and andesite) show general enrichment of light REEs (LREE) $((\mathrm{La} / \mathrm{Yb}) \mathrm{N})=4.4$ to 39.3$)$ and depletion of heavy REEs (HREE) $((\mathrm{Gd} / \mathrm{Yb}) \mathrm{N}$ $=0.79$ to 3.59$)$ (Figure 6$)$. Distinct weakly negative Eu anomaly $\left(\mathrm{Eu} /{ }^{\star} \mathrm{Eu}=0.65\right.$ to 0.98 ) of diorite, granodiorite, and granodiorite porphyry would be indicative of plagioclase fractionation [19]. Quartz feldspar porphyry, dacite and andesite porphyry characterized by negative to positive Eu anomalies $\left(\mathrm{Eu} /{ }^{\star} \mathrm{Eu}=1.15\right.$ to 2.41). Negative of Eu anomalies indicates possible fractionation of Eu to plagioclase, while the positive $\mathrm{Eu}$ anomalies suggest that Eu present in primary plagioclase are immobile during potassic alteration, while other REE were slightly removed during potassic alteration. Thus, REE patterns are slightly mobile during alteration. The primitive mantle-normalized trace elements diagram of intrusive and volcanic rocks in Halo are characterized by enrichments of large-ion lithophile elements (LILE, such as $\mathrm{K}, \mathrm{Rb}$ and $\mathrm{Ba}$ ) and depletions of high field strength elements (HFSE, such as $\mathrm{Nb}$ and $\mathrm{Ti}$ ) (Figure 6).

Quartz feldspar porphyry samples (QFP1-1 to QFP1-4) contain higher total REE concentration ranging from 44.8 to $54.6 \mathrm{ppm}$ compared to quartz feldspar porphyry samples (QFP1-5 to QFP1-10) which contain lower total REE concentration ranging from 16.4 to $35.9 \mathrm{ppm}$. Based on petrography, all quartz feldspar porphyries are potassicaly altered and samples QFP1-1 to QFP1-4 are less altered than the samples QFP1-5 to QFP1-10. This suggests that the REE was slightly removed during potassic alteration, except for Eu which was accommodated in plagioclase and immobile during potassic alteration. 

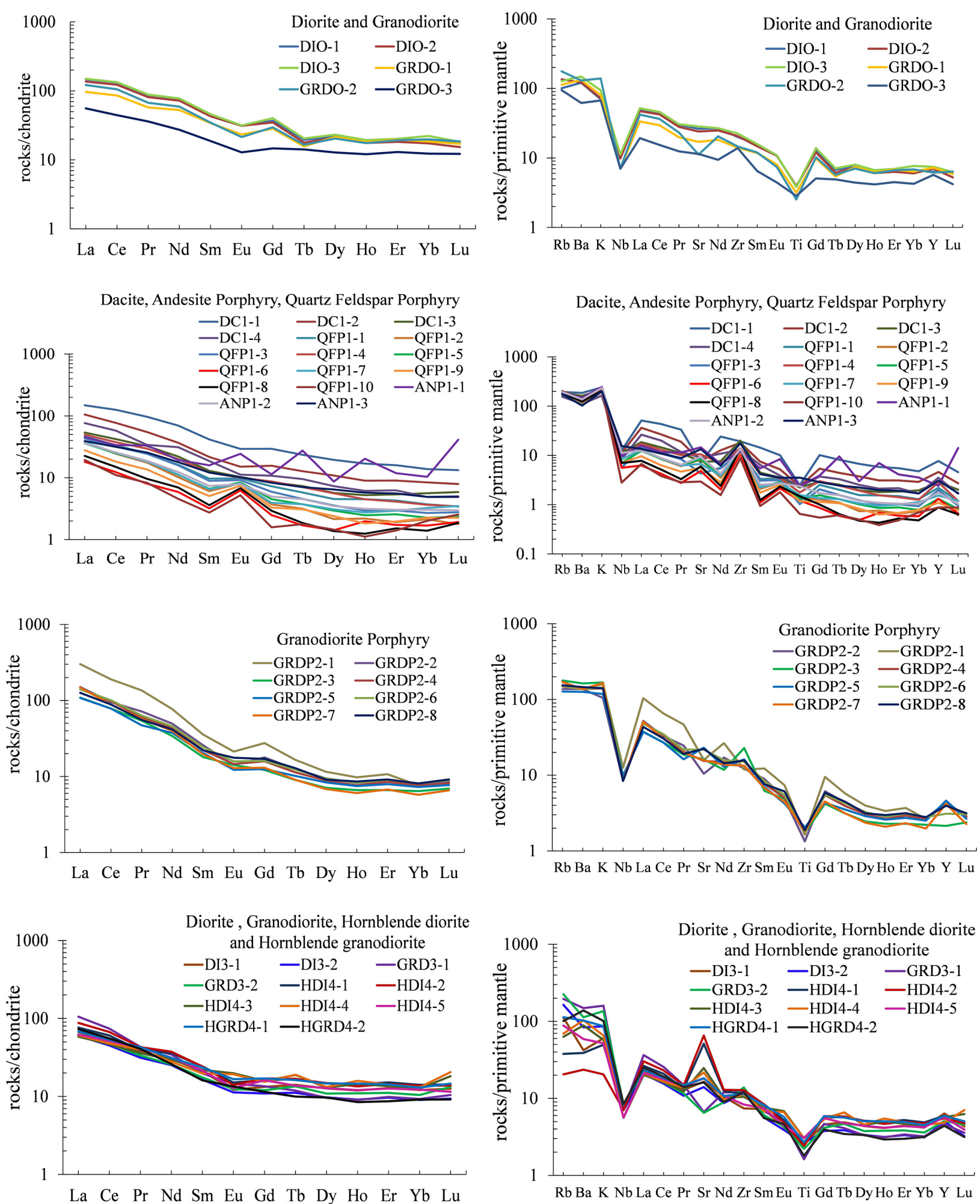

Figure 6. Chondrite-normalized rare earth element and primitive-mantle-normalized trace element patterns of intrusive and volcanic rocks in the Halo prospect [18]. Abbreviation: DIO-1 to DIO-3 and GRDO-1 to GRDO-3: diorite and granodiorite, respectively, exposed on surface; DC1-1 to DC1-4, ANP1-1 to ANP1-3, and QFP1-1 to QFP1-10: dacite, andesite porphyry and quartz feldspar porphyry, respectively, in drill hole HD1; GRD2-1 to GRD2-8: granodiorite porphyry in drill hole HD2; DI3-1 to DI3-2 and GRD3-1 to GRD3-2: diorite and granodiorite, respectively, in drill hole HD3; HDI4-1 to HDI4-5 and HGRD4-1 to HGRD4-2: hornblende diorite and hornblende granodiorite, respectively, in drill hole HD4. 


\section{Molar Element Ratios}

General element ratios (GERs) and Pearce element ratios (PERs) are both molar element ratios (MERs) used to depict geochemical processes such as alteration ([17] \& [20]). PER analysis uses molar element concentrations ratio to the molar concentration of an element which has remained unchanged during mass transfer processes, i.e. a "conserved" element ([21] \& [22]). Thus in order to quantify the intensity of hydrothermal alteration around major mineralized zones, it is necessary to use a technique that distinguish the metasomatic impact of the mineralizing fluids, a prerequisite for which is pre-existing geochemical heterogeneity in the host rocks. It is also necessary to discriminate between the effects of hydrothermal activity and unrelated events such as weathering or metamorphism. A conserved element must be identified for use as a denominator in the PERs. Conserved elements are also used to test the cogenetic character of the rocks and group them accordingly. A PER diagram of Pr versus Nd shows the same behavior during alteration and a best-fit line with a positive slop that intersects the origin indicating that the rocks are derived from a single precursor (Figure 7(a); [23]). The samples are cogenetic, thus PER can be adapted [22].

A PER plot of $(2 \mathrm{Ca}+\mathrm{Na}+\mathrm{K})$ versus $\mathrm{Al} / \mathrm{Nd}$ (Figure $7(\mathrm{~b}))$ discriminates between unaltered and hydrothermally altered felsic rocks. The least altered samples plot closer to the feldspar-plagioclase control line with slop of 1 from the origin while the completely sericitized samples plot closer to the line with slop of $1 / 3$ from the origin [24]. The dacite samples plot around and toward the muscovite control line with slop $1 / 3$, suggesting the dacite has been affected by quartz-sericite-pyrite (phyllic) alteration (Figure $7(\mathrm{~b})$ ) while the quartz feldspar porphyry, andesite porphyry, diorite and granodiorite samples plot between the plagioclase and the muscovite control lines, suggesting that these rocks have been affected by varying degree of sericite alteration. This is consistent with plagioclase replaced by sericite observed in petrography. Potassic alteration such as secondary biotite or secondary K-feldspar in rocks is not discriminated from unaltered rocks on this diagram. Epidote alteration is represented by the control line with slope $16 / 3$. The hornblende diorite samples plot between the epidote and plagioclase control lines consistent with petrography and XRD analysis.

\section{Discussion}

\subsection{Genetic Implication}

The enrichment of LILEs and depletion of $\mathrm{Nb}$ and $\mathrm{Ti}$ are characteristic feature of magmas generated in a subduction-related tectonic setting ([25] \& [26]). The low $\mathrm{Nb}$ depletion is typical of calc-alkaline magmatic rocks formed in subduction zone environments and may be regarded as an indicator of crustal involvement in magmatic process ([27] \& [28]). Moreover, in the $(\mathrm{Y}+\mathrm{Nb})$ versus $\mathrm{Rb}$, and $\mathrm{Y}$ versus $\mathrm{Nb}$ diagrams (Figure 8; [29]), samples plot within the field of volcanic arc granite. It is consistent with the lithochemistry of porphyry copper-(molybdenum-gold) deposits formed by magmatic-hydrothermal fluids generated from subduction related magmatism. 


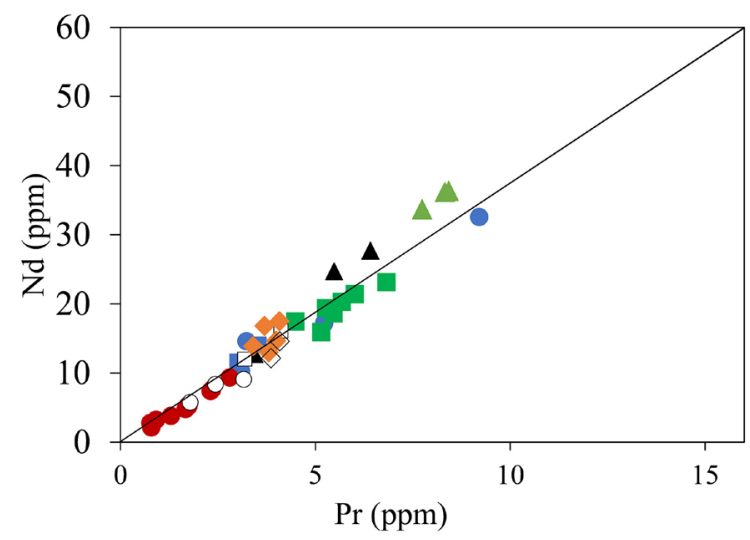

(a)

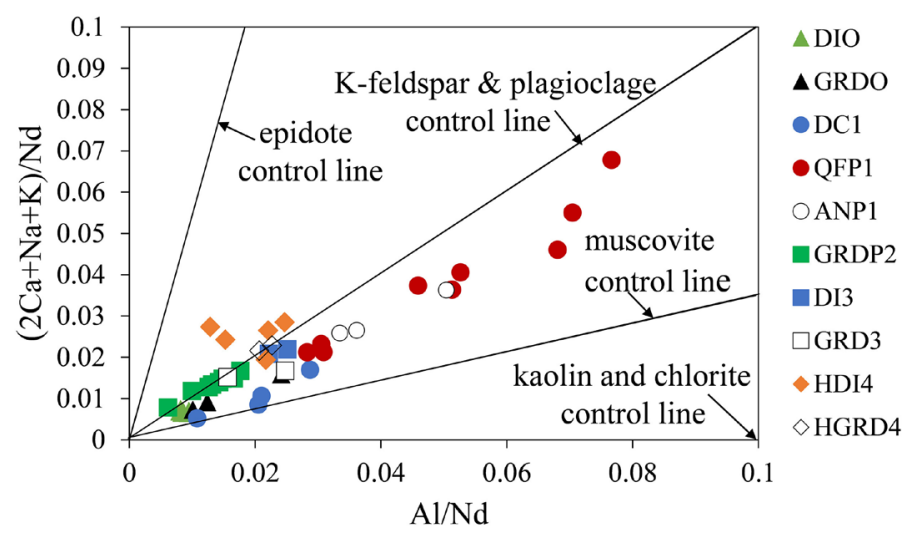

(b)

Figure 7. (a) PER diagram of Pr versus Nd of the least altered and altered samples in the Halo prospect showing the linear plot that indicated the samples are cogenetic; (b) PER diagram of $(2 \mathrm{Ca}+\mathrm{Na}+\mathrm{K}) / \mathrm{Nd}$ versus $\mathrm{Al} / \mathrm{Nd}$ to discriminates unaltered felsic samples from altered samples. All elements are expressed as molar concentrations except for Nd which is expressed in ppm. Symbols and abbreviations are the same as Figure 5.
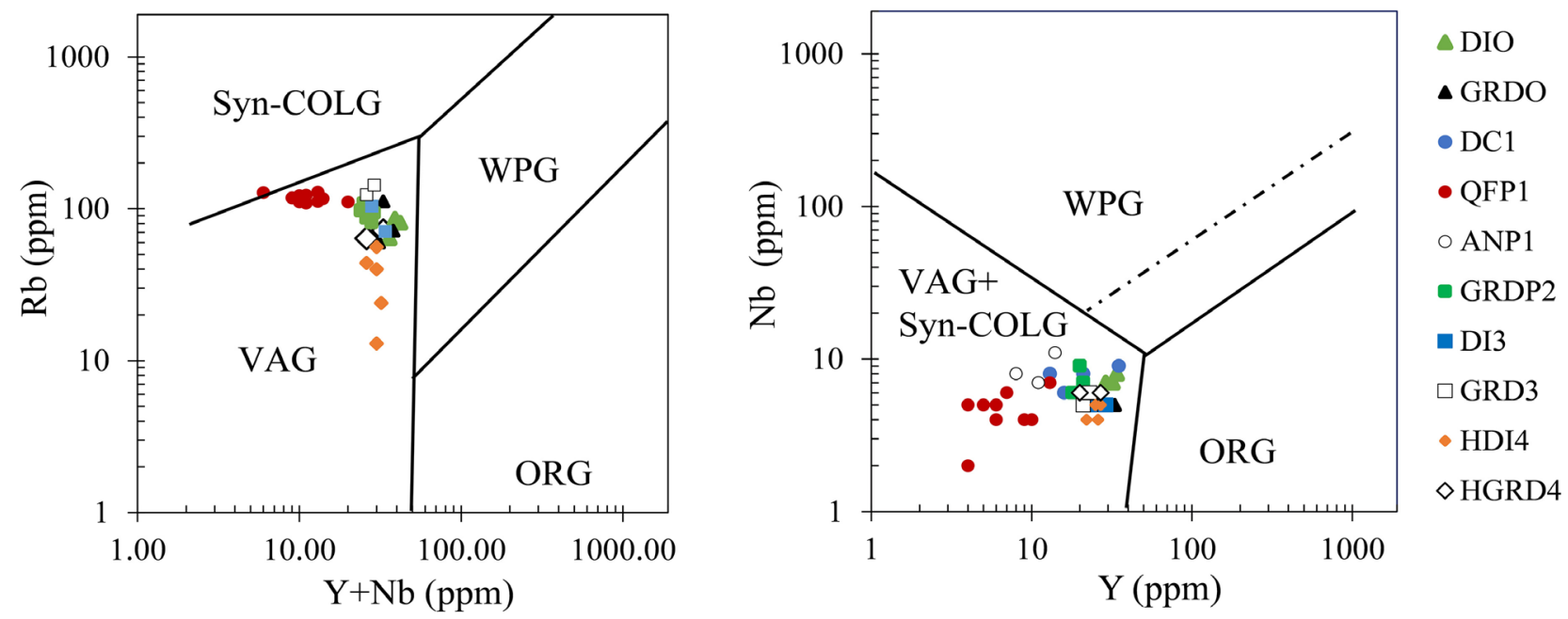

Figure 8. Tectonic discrimination diagram for plutonic rocks [29]. VAG: volcanic-arc granite, COLG: syn-collision granite, WPG: within plate granite, ORG: oceanic ridge granite. Symbols and abbreviations are the same as Figure 5.

\subsection{Tectonic Affinity}

The fresh and least-altered diorite and granodiorite were selected to discriminate between typically arc rocks and arc adakite. In the commonly used $\mathrm{Sr} / \mathrm{Y}$ versus $\mathrm{Y}$ diagram, the diorite and granodiorite dominantly plot in typically arc (Figure 9(a); [30]). This result is consistent with relatively unfractionated HREE patterns of the samples plotted in Figure 6, which implies that partial melting occurred at a depth above the garnet stability field that would be shallower than the subducted slab. Furthermore, based on the $(\mathrm{La} / \mathrm{Yb}) \mathrm{N}$ versus $\mathrm{YbN}$ diagram, the diorite and granodiorite plot in the field of post-Archean subduction-related granitoids (Figure 9(b); [31]). This is in the good agreement with the conclusion of [32] who assumed that the Southeast Asian margin was an Andean-type volcanic arc from mid-Jurassic to mid-Cretaceous. 


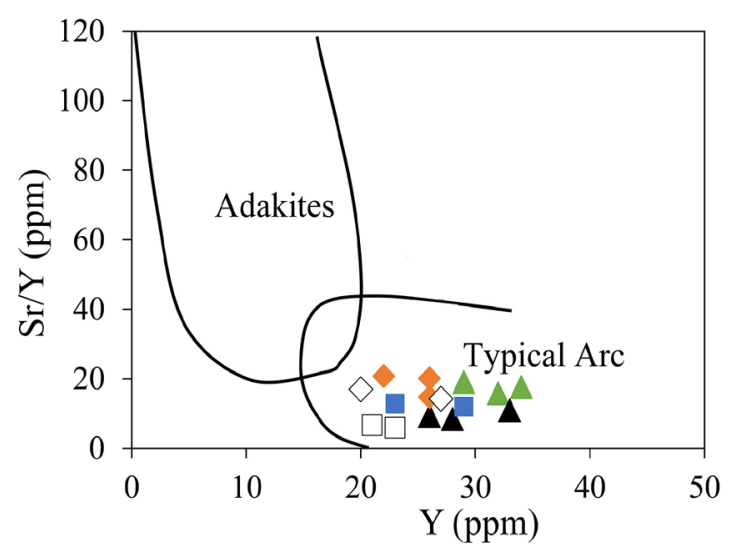

(a)



(b)

Figure 9. Discrimination diagram for the origin of intrusive rocks in the Halo prospect and (a) Plot Y (ppm) versus Sr/Y diagram with fields from [30]; (b) Plot of La/YbN versus YbN with fields from [31]. Symbols are the same as in Figure 4.

\subsection{Alteration Lithogeochemistry}

In the PER diagram $(2 \mathrm{Ca}+\mathrm{Na}+\mathrm{K}) / \mathrm{Nd}$ versus $\mathrm{Ca} / \mathrm{Nd}$ (Figure $7(\mathrm{~b})$ ), quartz feldspar porphyry and andesite porphyry are only moderately altered while dacite exhibits the highest alteration indices. Rock units with highest alteration are indicated by muscovite (sericite) alteration. Moreover, the behaviour of $\mathrm{K}$ during alteration can be broadly understood in the PER diagram of K/Al versus $(2 \mathrm{Ca}+\mathrm{Na}+\mathrm{K}) / \mathrm{Al}$ for felsic lithologies (Figure 10(b); [24]). These diagrams spatially provide the location of the main alteration minerals by discriminating between potassic, sericite and argillicalteration for felsic and intermediate lithologies. The least altered rocks plot closer to $(1,0)$ while potassic alteration mineral (K-feldspar and biotite) plot towards the $(1,1)$, muscovite plot at $(1 / 3$, $1 / 3)$ and kaolin group minerals and chlorite plot at $(0,0)$. General and Pearce element ratios can identify the three alteration in the Halo prospect such as potassic (secondary K-feldspar and biotite), phyllic (sericite) and propylitic (epidote) alteration. Results are also consistent with petrography (Figure 3, Figure 10).

Pearce Element Ratios (PERs) diagrams can discriminate between the effects of hydrothermal activity and unrelated events such as weathering or metamorphism. Moreover, these diagrams also can determine the alteration index (AI) by identifying the slop of the line connecting the sample point and its origin. AI is defined by dividing the ordinate value ( $y$-axis) with absicca value ( $\mathrm{x}$-axis) [24]. AI values were rescaled so that a value of 1 , representing AI of an unaltered samples as $0 \%$, and AI of totally altered rocks as $100 \%$. AI of the quartz feldspar porphyry and the andesite porphyry associated potassic alteration ranges from $11.8 \%$ to $32.3 \%$ and that of the dacite associated with phyllic alteration ranges from $43.3 \%$ to $66.6 \%$ while that of two samples of granodiorite porphyry ranges from $5.1 \%$ to $11.9 \%$. The highly altered zones are commonly vectoring towards the center of the hydrothermal system which can represent the locus of mineralization (e.g. [22] \& [24]). 


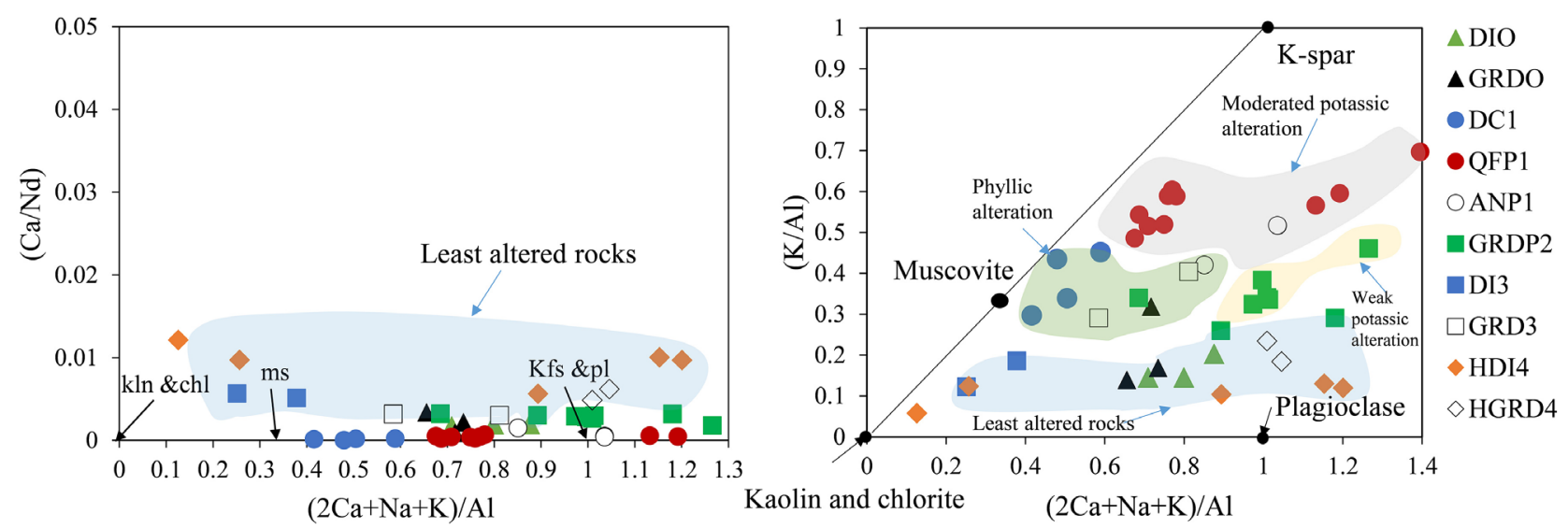

Figure 10. (a) PER diagrams showing the degree of alteration for felsic rocks. Samples with $x=1$ are considered as fresh while samples with $x=0$ are highly altered; (b) K/Al versus to alteration index (x-axis) to discriminate the alteration samples such as muscovite, K-feldspar, biotite, kaolin alteration minerals and chlorite with fresh rocks. Symbols and abbreviations are the same as Figure 5.

\section{Conclusions}

1) The Halo porphyry copper molybdenum prospect is hosted in diorite, granodiorite, quartz feldspar porphyry, granodiorite porphyry, andesite porphyry, mafic dykes, aplite dykes, and felsic dykes. Quartz feldspar porphyry and granodiorite porphyry are considered as the main intrusive body and most favorable host copper-molybdenum mineralization. They intersected drill hole HD1 and $\mathrm{HD} 2$, which are associated with potassic alteration and dominated by $\mathrm{Cu}-\mathrm{Fe}$ sulfide mineralization, quartz vein stockworks and magnetite veins.

2) The diorite and granodiorite belong to medium-K calc-alkaline to high- $\mathrm{K}$ calc-alkaline series while rock-series of volcanic rocks and quartz feldspar porphyries were not determined due to the mobility of $\mathrm{K}$ during alteration. The intrusive rocks in the Halo prospect display geochemical features of trace elements typical of magmatism in a subduction zone, such as enrichment of LILE and negative anomalies of $\mathrm{Nb}$ and $\mathrm{Ti}$. The intrusive rocks and volcanic rocks plot within the domains of volcanic arc granite based on spider diagram, $(\mathrm{Y}+\mathrm{Nb})$ versus $\mathrm{Rb}$ diagram, and $\mathrm{Y}$ versus $\mathrm{Nb}$ diagram. Lithochemistry of intrusive and volcanic rocks in the Halo porphyry copper-molybdenum prospect is consistent with the rocks associated with porphyry deposits type ore in the world generated by magmatism in subduction setting related.

3) General element ratios and Pearce element ratios (PER) define three alterations in the Halo prospect such as potassic (secondary K-feldspar and biotite), phyllic (sericite) and propylitic (epidote) alteration. The alteration index of the dacite and the quartz feldspar porphyry calculated from PER are higher than that of the granodiorite porphyry. This is consistent with the high copper concentration of the dacite and the quartz feldspar porphyry up to $7048 \mathrm{ppm}$. The highly altered zone vectoring center of the hydrothermal system may represent the locus of mineralization.

4) According to result mentioned above, the Halo prospect is a porphyry cop- 
per-molybdenum prospect and drill hole HD1 and HD2 are situated close to the centre of porphyry deposit than drill hole HD3 and HD4.

\section{Acknowledgements}

The authors wish to express our special thanks to Professor Akira Imai, Department of Earth Resources Engineering, Kyushu University in Japan for his kind support and encouragement to carry out this research work, advice and reading the manuscript and give facilities in varies ways. We would like to express our deepest thanks to Mr. John Dau Pau, a Vice President of Operation, Mr. Mike Weeks, a chairman in Angkor Gold, for allowing and supporting to the field research in the Halo prospect, Oyadao South area, Ratanakiri, Cambodia. We would like to thanks also for Craig T. Richardson for is excellent master thesis study made on PhumSyarung-Dok Yong Fault Corridor (Cambodia), and the exceptional cooperation of Dennis Ouellet (Exploration Manager of Angkor Gold Corp.). We would like to express our gratitude to the ASEAN University Network/Southeast Asia Engineering Education Development Network (AUN/SEED-NET), Japanese International Cooperation Agency (JICA) for the PhD scholarship. We would like to special thank JSPS KAKENHI Grants Number 18H01927 and JSPS Core-to-CoreProgram provide us financial support during field work. I am also indebted to all lecturers and Dr. NallisKry, a head of the Faculty of Geo-resources and Geotechnical Engineering, Institute of Technology of Cambodia, Cambodia, who has given me the constant support and guidance for my $\mathrm{PhD}$ at Kyushu University.

\section{Conflicts of Interest}

The authors declare no conflicts of interest regarding the publication of this paper.

\section{References}

[1] Angkor Gold Crop (2018) Report of the-Geological Overview of Angkor Gold Projects, Cambodia. 6-35. http://www.angkorgold.ca/wp-content/uploads/2014/06/GEO-PPT-March-2018.pdf

[2] Metcalfe, I. (1999) Gondwana Dispersion, Asian Accretion: An Overview. In: Matcalfe, I., Ed., Gondwana Dispersion and Asian Accretion, A.A. Balkema, Rotterdam, 9-28. https://doi.org/10.1080/08120099608728282

[3] Metcalfe, I. (2013) Gondwana Dispersion and Asian Accretion: Tectonic and Palaeogeographic Evolution of Eastern Tethys. Journal of Asian Earth Sciences, 66, $1-33$.

[4] KhinZaw, Meffre, S., Lai, C.K., Burrett, C., Santosh, M., Graham, I., Manaka, T., Salam, A., Kamvong, T. and Cromie, P. (2013) Tectonics and Metallogeny of Mainland Southeast Asia-A Review and Contribution. Gondwana Research, 26, 5-30. https://doi.org/10.1016/j.gr.2013.10.010

[5] Manaka, T., Zaw, K., Lim, Y. and Meffres, S. (2012) Update Geology and Tectonic Framework of Cambodia and South Vietnam. 1st Quarterly Report for Ore Deposits of SE Asia Project, 9-10. 
[6] Thuy, T.B.N., Satir, M., Siebel, W. and Chen, F. (2004) Granitoids in the Dalat Zone, Southern Vietnam: Age Constraints on Magmatism and Regional Geological Implications. International Journal of Earth Sciences, 93, 329-340. https://doi.org/10.1007/s00531-004-0387-6

[7] Zaw, K. and Meffre, S. (2010) Final Report on Ore Deposits of SE Asia Project.

[8] Banks, M. and Minerals, Z. (2008) Report of Stage One Exploration Results, TienThuan Project. Binh Dinh Province, Vietnam, 1-5.

[9] CAN, Indochine Mining Limited (2010) Report of Kratie Project and Ratanakiri Project in Cambodia, 23-27.

[10] Richardson, C.T., Craig, J.R., Adrian, M. and Kurtis, D. (2016) Controls on Polymetallic Vein Deposits and Porphyry Deposits in the PhumSyarung-Dok Yong Fault Corridor, Ratanakiri Province, Cambodia. Unpublished Poster, Society of Economic Geologists.

[11] Angkor Gold Crop (2017) Report of the New Copper Porphyry System and Gold Update on Drill Program at Oyadao South Halo Project. Cambodia, 1-3.

http://www.angkorgold.ca/angkor-discovers-new-copper-porphyry-system-oyadaosouth-halo-project/

[12] Berger, B.R., Ayuso, R.A., Wynn, J.C. and Seal, R.R. (2008) Preliminary Model of Porphyry Copper Deposits. US Geological Survey Open-File Report Geological Survey, Reston, VA. https://pubs.usgs.gov/of/2008/1321/pdf/OF081321_508.pdf https://doi.org/10.3133/ofr20081321

[13] Large, R.R., Gemmell, J.B., Paulick, H. and Huston, D.L. (2001) The Alteration Box Plot: A Simple Approach to Understanding the Relationships between Alteration Mineralogy and Lithogeochemistry Associated with VHMS Deposits. Economic Geology, 96, 957-971. https://pdfs.semanticscholar.org/7829/832df74b6e1a240fa3939f8f35ac40241f6f.pdf

[14] Cox, K.G., Bell, J.D. and Pankhurst, R.J. (1979) The Interpretation of Igneous Rocks. George Allen and Unwin Boston, London. https://doi.org/10.1007/978-94-017-3373-1

[15] Winchester, J.A. and Floyd, P.A. (1977) Geochemical Discrimination of Different Magma Series and their Differentiation Products Using Immobile Elements. Chemical Geology, 20, 325-343. https://doi.org/10.1016/0009-2541(77)90057-2

[16] Peccerillo, A. and Taylor, S.R. (1976) Geochemistry of Eocene Cal-Alkaline Volcanic Rocks from the Kastamonu Area, Northern Turkey. Contributions to Mineralogy and Petrology, 58, 63-81. https://doi.org/10.1007/BF00384745

[17] Pearce, T.H. (1968) A Contribution to the Theory of Variation Diagrams. Contribution to Mineral and Petrology, 19, 142-157. https://doi.org/10.1007/BF00635485

[18] Sun, S.S. and McDonough, W.F. (1989) Chemical and Isotopic Systematic of Oceanic Basalts: Implication for Mantle Composition and Processes. In: Saunder, A.D. and Norry, M.J., Eds., Magmatism in Ocean Basins, Geological Society, Special Publication, London, 313-345.

[19] Davidson, J., Turner, S. and Plank, T. (2013) Dy/Dy*: Variations Arising from Mantle Sources and Petrogenetic Processes. Journal of Petrology, 54, 525-537. https://doi.org/10.1093/petrology/egs076

[20] Stanley, C.R. and Russell, J.K. (1989) Petrologic Hypothesis Testing with Pearce Element Ratio Diagrams: Derivation of Diagram Axes. Contributions to Mineralogy and Petrology, 103, 78-79. https://doi.org/10.1007/BF00371366

[21] Stanley, C.R. and Madeisky, H.E. (1995) Lithogeochemical Exploration for Hydro- 
thermal Mineral Deposits Using Pearce Element Ratio Diagrams. In: Extended $A b$ stracts, Townsville, Queensland, 259-262.

[22] Stanley, C.R. and Madeisky, H.E. (1994) Lithogeochemical Exploration for Hydrothermal Ore Deposits Using Pearce Element Ratio Analysis. In: Lentz, D.R., Ed., Alteration and Alteration Processes Associated with Ore-Forming Systems, Waterloo, Canada, 193-212.

[23] MacLean, W.H. and Barrett, T.J. (1993) Lithogeochemical Techniques Using Immobile Elements. Journal of Geochemical Exploration, 48, 109-133. https://doi.org/10.1016/0375-6742(93)90002-4

[24] Urqueta, E., Clark, A.H., Stanley, C.R., Oates, C.J. and Kyser, T.K. (2009) Lithogeochemistry of the Collahuasi Porphyry $\mathrm{Cu}-\mathrm{Mo}$ and Epithermal $\mathrm{Cu}-\mathrm{Ag}$ (-Au) Cluster, Northern Chile: Pearce Element Ratio Vectors to Ore. Geochemistry: Exploration, Environment, Analysis, 9, 9-17. https://doi.org/10.1144/1467-7873/07-169

[25] Wood, D.A., Joron, J.L. and Treuil, M. (1979) A Reappraisal of the Use Trace Elements to Classify and Discriminate between Magma Series Erupted in Different Tectonic Settings. Earth and Planetary Science Letters, 68, 297-308.

[26] Briqueu, L., Bougault, H. and Joron, J.L. (1984) Quantification of Nb, Ta, Ti and V Anomalies in Magmas Associated with Subduction Zones-Petrogenetic Implications. Earth and Planetary Science Letters, 68, 297-308. https://doi.org/10.1016/0012-821X(84)90161-4

[27] Rollinson, H.R. (1993) Using Geochemical Data: Evaluation, Presentation, and Interpretation. Longman Scientific and Technical, Singapore, $352 \mathrm{p}$.

[28] Lan, C.Y.J., Jahn, B.M., Mertzman, S.A. and Wu, T.W. (1996) Subduction-Related Granitic Rocks of Taiwan. Journal Southeast Asian Earth Science, 14, 11-28. https://doi.org/10.1016/S0743-9547(96)00017-7

[29] Pearce, J.A., Harris, N.B.W. and Tindle, A.G. (1984) Trace Element Discrimination Diagrams for the Tectonic Interpretation of Granitic Rock. Journal of Petrology, 25, 956-983. https://doi.org/10.1093/petrology/25.4.956

[30] Defant, M.J. and Drummond, M.S. (1990) Derivation of Some Modern Magmas by Melting of Young Subducted Lithosphere. Nature, 347, 662-665. https://doi.org/10.1038/347662a0

[31] Martin, H. (1986) Effect of Steeper Archean Geothermal Gradient on Geochemistry of Subduction-Zone Magmas. Geology, 14, 753-756. https://doi.org/10.1130/0091-7613(1986)14<753:EOSAGG>2.0.CO;2

[32] Taylor, B. and Hayes, D.E. (1983) Origin and History of the South China Sea Basin. In: Hayes, D.E., Ed., The Tectonics and Geologic Evolution Southeast Asian Seas and Islands, Part 2, Geophysical Monograph 27, American Geophysic Union, Washington DC, 23-56. https://doi.org/10.1029/GM027p0023 